2 Title: Bone Marrow-Derived Cells Contribute to the Maintenance of Thymic Stroma including

3 Thymic Epithelial Cells.

4 Running Title: Bone marrow derived CD45+EpCAM+cells Contribute to TECs

5 Authors: Shami Chakrabarti**; Mohammed Hoque*; Nawshin Zara Jamil"; Varan J Singh",

6 Neelab Meer ${ }^{\sharp}$, Mark T. Pezzano ${ }^{\# \S}$

7

8 Affiliation: *PhD Program in Biochemistry, The Graduate Center of the City University of

$9 \quad$ New York

10 \#Department of Biology, City College of New York CUNY

$11 \S$ PhD Program in Biology, The Graduate Center of the City University of New York

12 Corresponding Author: Mark T Pezzano

13

Email: $\quad$ mpezzano@ccny.cuny.edu

14

Phone: $\quad$ 212-650-8574 (Office) 8577 (Lab)

15

Fax:

212-650-8585

16

17

18 Acknowledgements:

19 This work was supported by NIH/NIAID 9SC1AI04994, NIH-

20 NCI U54CA137788/U54CA132378, NIMHHD 8G12MD007603. We would like to thank

21 Jeffrey Walker for his flow cytometry support.

22 
23 Abstract:

25 In paradox to critical functions for T-cell selection and self-tolerance, the thymus undergoes

26 profound age-associated atrophy and loss of T-cell function, which are further enhanced by

27 cancer therapies. Identification of thymic epithelial progenitor populations capable of forming

28 functional thymic tissue will be critical in understanding thymic epithelial cell (TEC) ontogeny

29 and designing strategies to reverse involution. We identified a new population of progenitor

30 cells, present in both thymus and bone marrow (BM), that co-express the hematopoietic marker

31 CD45 and the definitive thymic epithelial marker EpCAM and maintains the capacity to form

32 functional thymic tissue. Confocal analysis and qRT-PCR of sorted cells from both BM and

33 thymus confirmed co-expression of CD45 and EpCAM. Grafting of C57BL/6 fetal thymi under

34 the kidney capsule of H2BGFP transgenic mice revealed that peripheral CD45+ EpCAM+ GFP-

35 expressing cells migrate into the developing thymus and contribute to both TECs and FSP1 -

36 expressing stroma. Sorted BM-derived CD45+EpCAM+ cells contribute to reaggregate thymic

37 organ cultures (RTOCs) and differentiate into keratin and FoxN1 expressing TECs,

38 demonstrating that BM cells can contribute to the maintenance of TEC microenvironments

39 previously thought to be derived solely from endoderm. BM-derived CD45+EpCAM+ cells

40 represent a new source of progenitor cells that contribute to thymic homeostasis. Future studies

41 will characterize the contribution of BM-derived CD45+EpCAM+ TEC progenitors to distinct

42 functional TEC microenvironments in both the steady-state thymus and under conditions of

43 demand. Cell therapies utilizing this population may prove useful for counteracting thymic

44 involution in cancer patients. 
46 Introduction:

47 The thymus serves as the primary lymphoid organ responsible for the development and

48 selection of a self-tolerant T-cell repertoire $(1,2)$. Thymic epithelial cells (TECs) are the most

49 significant component of the thymic microenvironment responsible for regulating $\mathrm{T}$ cell

50 development and selection(2-4). Thus, proper organization and maintenance of TECs are critical

51 for a properly functioning adaptive immune $\operatorname{system}(2,3,5)$. Despite the fundamental

52 importance of the thymus in the development of T cells, the thymus undergoes profound

53 atrophy relatively early in life(6-8). Thymus degeneration is physically evident in humans

54 starting at puberty, where the loss of functional thymic microenvironments contributes to a

55 progressively restricted naïve $\mathrm{T}$ cell repertoire, resulting in an immune system that is less

56 capable of responding to new immune challenges $(6,9)$. Thymic involution also severely

57 restricts the ability to generate long term, low morbidity tolerance to foreign transplants,

58 including those of stem cell origin(10). Understanding the signals and cell-to-cell interactions

59 that control both the development and postnatal maintenance of thymic epithelial cells is critical

60 to design clinical strategies to counteract age-associated involution while enhancing thymic

61 recovery following HSCT.

62

63 Structurally, the thymic stroma contains three compartments: 1) Cortex, 2) Medulla, and 3)

64 Cortico-medullary junction(11). The cortex is composed of cortical thymic epithelial cells

65 (cTEC), defined by primarily keratin 8 expression as well as the surface expression of BP1 and

66 CD205, together with immature thymocyte subsets and it is involved in positive selection and

67 MHC restriction during T-cell development(12). The thymic medulla is composed of medullary

68 thymic epithelial cells (mTEC), defined by primarily Keratin 5/Keratin 14 expression together 
69 with UEA1 binding, and is involved in negative selection of mature single-positive (SP)

70 thymocytes expressing autoreactive T-cell antigen receptors and development of

71 FoxP3+Tregs $(12,13)$. The cortico-medullary junction is the perivascular space between the

72 cortex and medulla, which serves as the entry point of CD4-CD8-(DN) thymocytes to the

73 thymus(14) as well as the location where fully mature SP naïve T-cells exit to the peripheral

74 blood vessels $(2,5,12)$.

75

76 Most epithelial tissues are known to maintain tissue homeostasis throughout their adult life

77 through the action of progenitor or stem cell populations(15). Previous work has shown that

78 TECs of both the cortex and medulla develop from common progenitors during thymic

79 organogenesis (16-19). The presence of progenitors restricted to each lineage has also been

80 demonstrated(16, 20-24). Hamazaki et al., 2007 identified embryonic TEC progenitors,

81 expressing high levels of claudin-3 and claudin-4, that exclusively give rise to mature

82 mTECs(20). Progenitors initially expressing cTEC lineage markers(25), including the

83 thymoproteosome subunit $\mathrm{b} 5 \mathrm{t}(26,27)$ and CD205(19), were shown to give rise to mTECS

84 during thymic organogenesis. However, the mechanisms responsible for the maintenance of

85 functional thymic microenvironments in the postnatal thymus remain unclear. Tissue

86 maintenance might be mediated through stem-cell-based regeneration similar to tissues with

87 high turnover rates, including hematopoietic, skin, and intestinal tissues(28). Alternatively, the

88 thymus might be maintained by replication of more differentiated cells, similar to tissues with

89 lower turnover rates, including the liver and pancreas(29). Gray et al. 2006 revealed that TECs,

90 particularly mTECs, have much higher turnover rates than previously thought and are

91 comparable with keratinocytes(30), implying a stem-cell-based regeneration of at least mTECs. 
92 Sekai et al. 2014 identified embryonic SSEA-1+ Cld3,4hi mTEC stem cells that could maintain

93 functional mTEC regeneration, including mature mTECs and central T cell tolerance(31). The

94 clonogenic activity these SSEA-1+ Cld3,4hi TECs was rapidly decreased after birth in wild-

95 type (WT) mice but was maintaining in postnatal Rag2 deficient mice, suggesting that crosstalk

96 previously thought to be important in maintaining an appropriately organized thymus might

97 inhibit maintenance of mTEC stem cell activity(31).

98 The existence of bipotent TEPC in the postnatal thymus has also been demonstrated(16, 23, 32,

9933 ), and they appear to be found within the cTEC population(34)The stem cell characteristics of

100 label retention and increased colony-forming capacity were identified within the Sca-1hi

101 MHCIIlo TEC subset(32, 33). In contrast, Ulyanchenko et al. demonstrated bipotent TEPC

102 potential within the PLET1+ MHCIIhi subset(23). These competing results suggest that more

103 work is needed to identify the bipotent epithelial progenitor phenotype and the mechanisms

104 responsible for postnatal TEC maintenance.

105

106 Hematopoietic stem cells (HSCs) give rise to a variety of hematopoietic cells found in the

107 thymus, including thymocytes, B cells, dendritic cells, and macrophages. Several recent studies

108 identified unexpected plasticity of bone marrow-derived hematopoietic stem cell

109 populations(35-40). These studies suggested that bone marrow-derived HSC can give rise to a

110 variety of different adult cell types. Krause et al. showed that BM-derived HSC when

111 transplanted to irradiated hosts, home to and repopulate the bone marrow(35). They also

112 showed that this cell population could migrate and differentiate into epithelial cells in the lung,

113 GI tract, and skin(35). Theise et al showed, BM-derived HSC can give rise to hepatocytes in

114 both rodents and humans $(41,42)$. However, these studies were refuted by showing that BM- 
115 derived populations begin expressing characteristics of non-hematopoietic populations as a

116 result of cell fusion rather than by trans-differentiation in the hepatocyte system(43, 44). Later,

117 Borue et al. used a Cre-lox system to demonstrate that even though a small subset of stem cells

118 in the presence of somatic cells undergoes spontaneous fusion, there is a part of the HSC

119 population that can undergo trans-differentiation to contribute to the keratinocyte expressing

120 epithelial cells in the skin during the process of wound healing(45). They showed that BM-

121 derived epithelial cells at the wound edges express Ki67, proving that these trans-differentiated

122 cells are actively cycling(45). Wong et al. also showed that bone marrow-derived HSC could

123 give rise to lung epithelial cells under conditions of naphthalene induced lung injury(40).

124 Together, these studies showed unexpected plasticity, with BM-derived HSC giving rise to

125 epithelial cells in different organs, but none of these studies suggested a contribution of BM-

126 derived HSC to the maintenance of thymic epithelial or other thymic stromal components.

128 In this study, we describe a unique population of CD45+EpCAM+ cells in both adult BM and

129 thymus that expresses both the definitive hematopoietic marker CD45 and the definitive thymic

130 epithelial marker EpCAM. Using both organ transplant and reconstitution thymus organ

131 culture, we show that this unique population of cells can contribute to the maintenance of non-

132 hematopoietic components of thymic stroma, including keratin and FoxN1-expressing TECs. 
134 Materials and Methods:

$135 \quad$ Mice

136 C57BL6 and Actin H2BGFP and mRFP Rosa26 transgenic mice purchased from Jackson

137 Laboratory were used for this study. The ages of the mice ranged from 7-9 weeks. All mice

138 were bred and maintained at the City College of New York animal facility, and all experiments

139 were performed with approval from the City College of New York institutional animal care and 140 use committee.

$142 \quad$ Antibodies

143 The following primary antibodies were used for experiments: CD45-PE Cy7, CD45-APC Cy7, 144 CD45 APC (clone 30-F11, BD Bioscience), Pan-keratin ( Polyclonal, Dako), EpCAM-PE 145 (clone G8.8, eBioscience),FoxN1(clone G-20, Santa Cruz Biotechnology) FSP1(CloneS100A4, 146 Biolegend), Thy1.2(clone 30-H12, BD Bioscience), CD11b ( clone, BioLegend), CD11b 147 PerCpcy5.5 (clone M1/70, Biolegend) CD4- biotinylated(clone RM4-5, BioLegend), CD8 148 biotinylated (clone53-6.7, BioLegend), CD19 (clone1D3, Biolegend), Rabbit anti-GFP (Life), , 149 and CD205 (LY75/DEC-205)( clone HD30 (Millipore)'We also used lineage depletion panel 150 containing B220

151 The following secondary reagents were used for experiments: donkey anti-rabbit IgG-TRITC, 152 donkey anti-rabbit IgG-Cy5, donkey anti-rabbit IgG-FITC, donkey anti-rat IgG-TRITC, donkey 153 anti-goat IgG-FITC, goat anti-rat IgM-TRITC (Jackson ImmunoResearch), anti-rat IgG2a154 FITC, anti-rat IgM-FITC, streptavidin-APC, streptavidin-APC Cy7, streptavidin-PerCP Cy5.5 155 (BD Bioscience) and streptavidin-TRITC (Southern Biotechnology Associate). 


\section{TaqMan probes:}

158 All Taqman probes were purchased from Thermo-fisher Scientific, including CD45 (PTPRC), 159 EpCAM, and 18S rRNA.

160 Preparation of Thymic epithelial cells (TEC):

161 Thymic lobes were extracted from humanely euthanized mice in ice-cold phosphate-buffered

162 saline (PBS). The thymic lobes were cleaned to remove fat and connective tissue and cut into 163 four smaller pieces and transferred into a new tube containing PBS. The cut pieces were gently

164 agitated using a glass Pasteur pipette to remove thymocytes. The remaining tissue was

165 dissociated using Collagenase D $(1.8 \mathrm{mg} / \mathrm{ml})$ and DNase I (1X) in HBSS solution incubated in

166 a $37^{\circ} \mathrm{C}$ water bath for 15 minutes with gentle agitation every 5 minutes using a Pasture pipette.

167 The single-cell suspension of digested tissue was washed with PBS and then filtered through a

$168100 \mu \mathrm{m}$ strainer to remove any clumps of tissue. Hematopoietic cells were removed from the

169 single-cell suspension using anti-mouse Thy1.2, CD11b, and CD19 antibodies and magnetic

170 beads to enrich for the TEC population before sorting or flowcytometric analysis.

172 Isolation of Bone Marrow cells

173 Bone Marrow cells were isolated from mouse hind limbs, as previously described(46). The

174 resulting single-cell suspension was lineage depleted using biotinylated CD19, CD4, CD8,

175 CD11b, CD11c, B220, Ter119 antibody, and biotin-binder magnetic beads. The post-depletion 176 cell population was used for flow cytometric analysis. 
Flow cytometry

181 Cells were suspended in $100 \mu \mathrm{l}$ of FACS staining buffer (FSB-5\% fetal bovine serum, $5 \mathrm{mM}$

182 EDTA in PBS) with appropriately diluted primary antibodies for 20 minutes on ice in the dark.

183 After washing, secondary antibodies appropriately diluted in FSB were added, and the cells

184 were incubated for an additional 20 minutes on ice in the dark. After washing, the cells were

185 resuspended in $500 \mu \mathrm{l}$ of FSB for data acquisition. Data acquisition was performed using an

186 LSRII analyzer complete with three lasers (BD Bioscience), and cell sorts were performed using

187 a FACS Aria (BD Bioscience). FACS data were analyzed using Flow Jo software (Tree Star) or

188 FACS Diva software (BD Bioscience).

190 RNA extraction and q-RT PCR from sorted cells

191 Cells were sorted as described above, and RNA was isolated using the Trizol method as directed

192 by the Qiagen RNA isolation mini-prep kit. Isolated RNA was used for cDNA synthesis using

193 Reverse transcriptase and random hexamers. The resulting cDNAs were used for q-RT PCR

194 analysis using Taqman probes for CD45 and EpCAM. The 18s RNA housekeeping gene was

195 used as a positive control to normalize the qPCR results.

197 Reaggregate Thymic Organ Cultures (RTOC)

198 EpCAM+CD45+ cells were isolated and sorted to greater than $95 \%$ purity from the bone

199 marrow, and thymus-derived from Actin H2BGFP mice and placed in single-cell suspensions as

200 described above. The resulting highly purified EpCAM+CD45+ GFP+ cells were mixed with

201 dissociated fetal thymic stromal cells from an E14.5 C57BL6 fetal thymus at a ratio of 1:10 to

202 form a reaggregate on a polycarbonate filter suspended over a trans-well plate. After 48 hours 
203 of culture, these reaggregates were then surgically transplanted under the kidney capsule of

204 athymic Nude mice and grown for three weeks in vivo. After three weeks, the reaggregates were

205 harvested and sectioned for further IHC analysis.

206 Organ transfer experiments

207

208 Fetal thymi were isolated from E14.5 C57BL6 or Actin H2BGFP mice, as stated, and 5-6 fetal

209 lobes were placed under the kidney capsule of either Actin H2BGFP mice or Rosa26 mRFP

210 mice, respectively. Following a time course of 3, 6, 9, or 12 weeks the kidneys were isolated,

211 and most of the exogenous thymi were used for flow cytometric analysis, while the remaining

212 lobes were used for immunohistochemistry.

213

214 Immunohistochemistry of Thymic Sections

215 Thymi were embedded in OCT compound from Tissue-Tek and snap-frozen using Liquid-N2. 8

$216 \mathrm{~mm}$ sections were cut using a cryostat and mounted on coated slides. The slides were then fixed

217 in $4 \%$ paraformaldehyde for 10 minutes, washed with PBS three times, then permeabilized

218 using acetone for 10 minutes, washed and blocked with complete normal donkey serum (1\%

219 Normal donkey serum in Bovine serum albumin). Appropriately diluted antibodies specific to

220 stromal subsets were added to the slides and then placed into a humid chamber at $4^{\circ} \mathrm{C}$ overnight.

221 Secondary antibodies were applied, if needed, and incubated in a humid chamber for one hour

222 at $37^{\circ} \mathrm{C}$. After incubation, the slides were washed in PBS three times, mounted, then they were

223 sealed following the application of Prolong Gold antifade +DAPI reagent. Images were

224 acquired using a Zeiss LSM800 confocal microscope and LSM710 confocal microscope.

225 Confocal images were analyzed using analyzed using LSM software (Zeiss). 
227 Statistical Analysis

228 Data comparisons were performed using a non-parametric, unpaired, one-tailed T-test. All

229 graphs and statistical results were generated using Graph-pad Prism software. A P-value of

$230<0.01$ was considered significant.

231 


\section{Results:}

233 Cells co-expressing both the definitive thymic epithelial marker EpCAM and the hematopoietic

234 marker CD45 are present in both the bone marrow and the thymus.

235

236 Presence of CD45+EpCAM+ cells in the thymus:

237 Using standard enzymatic digestion of thymic tissue in conjunction with CD45 magnetic bead

238 depletion to reduce the frequency of hematopoietic cells, we observed a persistent but rare

239 population of cells that co-expressed both CD45+ and EpCAM+ (DP), shown in Figure 1A.

240 This population has been described in several publications; however, they were identified as

241 thymic nurse cells, which are complexes of cTECs with internalized CD45+ thymocytes(47).

242 Due to the consistent presence of this DP population, even after very conservative doublet

243 discrimination, we set out to determine if this unusual population truly represented thymic nurse

244 cells or was a unique previously undescribed cell population expressing both CD45 and

245 EpCAM. We enhanced the isolation of this population by depleting hematopoietic cells in our

246 enzymatically dissociated thymic tissue using a cocktail of antibodies, including anti-Thy 1.2,

247 CD11b, CD11c, and CD19 together, rather than the usual an anti-CD45 antibody. Figure 1

248 shows a representative FACS profile demonstrating the increase in the frequency of the

249 CD45+EpCAM+ population when anti-CD45 depletion was not utilized to deplete

250 hematopoietic cells. Panel A shows that when anti-CD45 depletion was used to analyze the

251 frequency of DP cells present in 8-month old C57BL/6 mice, the CD45+EpCAM+ population

252 represents only an average of $0.23 \%(+/-0.2 \%)$ of the total cells present, whereas in panel B

253 when a cocktail of anti- Thy1.2, CD11b, CD11c, and CD19 was used for depletion, the 
254 frequency of the CD45+EpCAM+ population in the total dissociated thymic cells increased to

255 an average of $1.14 \%$. (+/-0.3\%). Every set of experiments had four replicates.

256

257 Thymic CD45+EpCAM+ cells co-express both CD45 and EpCAM at both the protein and 258 mRNA level:

259 We reasoned that the apparent co-expression of CD45 and EpCAM, observed using flow

260 cytometry of dissociated thymic tissue, could be the result of epitope sharing caused by the

261 close association of thymocytes with thymic epithelial cells or due to penetration of CD45

262 antibodies into partially damaged TNC complexes. To confirm the presence of single cells co-

263 expressing both CD45 and EpCAM, CD45+EpCAM+ cells were sorted to high purity ( 95\%

264 purity) using a FACS sorter from dissociated thymic tissue using conservative doublet

265 discrimination. Cells were also gated to exclude hematopoietic cells using a cocktail of anti-

266 Thy1.2, CD11b, CD11c, and CD19. To confirm that the DP cells expressed both the CD45 and

267 EpCAM genes at the RNA level, RNA was isolated from CD45+EpCAM+; CD45+; EpCAM+;

268 cell populations sorted from thymic tissue with greater than $95 \%$ purity and used for RNA

269 isolation. All RNA samples were normalized to $5 \mathrm{ng} / \mu \mathrm{l}$ before performing qPCR. 18s rRNA

270 was used as a housekeeping gene in order to compare relative amounts of RNA expression (Fig.

271 2B-C). Isolated and normalized RNA was analyzed using qRT-PCR with Taqman primers

272 specific to EpCAM and CD45. Relative levels of RNA expression of both CD45 and EpCAM

273 were normalized in each sorted population to the levels of 18 s rRNA, to thoroughly rule out

274 epitope sharing as the cause of this unique population. Figure 3 shows the fold change in the

275 expression level for both CD45 and EpCAM between the two control populations

276 (CD45+EpCAM-and CD45-EpCAM+) and the CD45+EpCAM+ population. Panel A shows the 
277 FACS representation of the sorted populations. Panel B shows the relative CD45 expression in

278 all three populations, where the CD45+EpCAM- population serves as the positive control. As

279 expected, the CD45+ control population has a high level of CD45 expression, whereas the

280 EpCAM+ population has negligible ( 96-fold lower) CD45 expression compared to the CD45

281 only population. The DP population expressed 50 fold more CD45 than the CD45-EpCAM+

282 TEC population but had a 55-fold lower expression of CD45 than the CD45+ EpCAM-

283 population. Panel C shows the relative EpCAM expression in all three populations, where the

284 CD45-EpCAM+ population was used as a positive control. DP cells exhibited $\sim 52$-fold higher

285 EpCAM expression than the CD45+ population, while expression of EpCAM was $\sim 55 \%$ lower

286 than that observed in the EpCAM only TEC positive control. As expected, the CD45 only

287 population has negligible EpCAM expression ( 96 fold lower). The qRT-PCR confirmed that

288 the DP population co-expressed both CD45 and EpCAM mRNA.

289 Expression of both the CD45 and EpCAM surface proteins by sorted CD45+EpCAM+ cells

290 was verified using immunohistochemistry. Cells that were either CD45+ EpCAM- or CD45-

291 EpCAM+ were also sorted as controls and viewed under a fluorescence microscope to ensure

292 that the antibodies were specific and that the CD45+EpCAM+ cells observed using flow

293 cytometry were indeed single cells co-expressing both CD45 and EpCAM surface proteins.

294 Figure 2 (D-R) shows the Immunohistochemistry (IHC) results obtained using the sorted

295 CD45+EpCAM+ population and the control CD45+ EpCAM- or CD45-EpCAM+ populations

296 from C57BL6 thymus following Anti-CD45 antibody and anti-EpCAM antibody staining.

297 Combined CD11b, CD11c, and CD19 staining was performed on all sorted populations to rule

298 out Langerhans cells that are known to co-express $\operatorname{EpCAM(48-50)~as~well~as~to~rule~out~non-~}$

299 specific binding of the anti-CD45 and anti-EpCAM antibodies to FC receptors on hematopoietic 
cells, leading to a false-positive result. The CD45+EpCAM- control cells showed staining with only the anti-CD45 antibody (Fig. 2 D-H). The CD45-EpCAM+ control cells only exhibited anti-EpCAM staining (Fig. 2 I-M). However, the CD45+EpCAM+ cells stained strongly with both the anti-CD45 and anti-EpCAM antibodies while no staining with a cocktail of antiCD11b, CD11c, CD19 antibodies was observed (Fig.2N-R) and confirming that the staining

305 observed was not the result of non-specific Fc-receptor binding. The inset of the merged image

306 of the sorted CD45+EpCAM+ population shows a single representative cell expressing both

307 CD45 and EpCAM. IHC results of the sorted cells confirmed protein co-expression of both 308 CD45 and EpCAM on single cells.

309 To provide further evidence for the presence of this unique population and to localize the cells 310 within the thymus, we sought to determine if the DP population could be seen within sections of 311 an intact adult mouse thymus. Within adult mouse thymus, CD45+ cells far outnumber and are 312 close to TECs. To better visualize the rare DP cells, within the background of abundant CD45+ 313 thymocytes, we performed intraperitoneal injections of dexamethasone on 6-week-old C57BL/6 314 mice to specifically reduce the number of double-positive thymocytes, the most abundant 315 CD45+ cells in the thymus. Three days following the dexamethasone injections, when the 316 number of thymocytes is most reduced, the mice were sacrificed, and their thymi were 317 harvested, sectioned, and stained with antibodies specific to CD45 (hematopoietic) and Pan318 Keratin (epithelial) to enable identification of thymic stromal cells co-expressing CD45 and 319 EpCAM. Confocal imaging of the stained sections revealed rare cells that share both 320 hematopoietic (CD45+) and epithelial (Pan Cytokeratin) characteristics in the intact thymus 321 (supplemental Figure 1). Together the IHC and qRT-PCR results obtained with highly purified 322 sorted cells from mouse thymus and IHC of sections of intact thymus confirmed that the 
323 CD45+EpCAM+ cell population observed in dissociated thymic preparations truly co-express

324 CD45 and EpCAM at both the protein and mRNA levels. Further, these results suggest that we

325 and others in the field may be losing a large percentage of EpCAM+ cells by ignoring this

326 CD45+ EpCAM+ population, including potential TEC progenitors.

328 Rare CD45+ EpCAM+ Cells are present in the bone marrow.

329 Bone marrow-derived cells, including hematopoietic stem cells (HSCs), were previously

330 reported as contributors of epithelial cells for epithelial organs, including lung(40), gut, and

331 uterus through the process of trans-differentiation(35). Wong et al. showed that the BM-derived

$332 \mathrm{CD} 45+\mathrm{CCSP}+$ cell population contributes to the alveolar epithelial cell population (CCSP is a

333 unique alveolar epithelial cell marker)(39, 40). As the DP population is expressing the

334 hematopoietic marker CD45, we wanted to determine if the CD45+EpCAM+ cells observed in

335 the thymus might be derived from a population in the BM. Bone marrow preparations were

336 analyzed for the presence of CD45+ EpCAM+ cells using flow cytometry. Multiple

337 experiments with C57BL6 bone marrow showed that bone marrow contains a low frequency

$338(\sim 0.1 \%-0.2 \%)$ of CD45+EpCAM+ cells (Figure 3A). To confirm that the population of CD45+

339 EpCAM+ cells detected in flow cytometry analysis actual represented single cells co-expressing

340 both the CD45 and EpCAM proteins on their surface, and also expressed in mRNA level,we

341 performed the same set of experiments shown above for the thymic CD45+EpCAM+ cell

342 population. CD45+EpCAM+ cells and CD45+EpCAM-cells (control) were sorted to high purity

$343(\sim 95 \%)$ from a bone marrow preparation depleted of lineage-committed cells and stained with

344 CD45 and EpCAM antibodies. The sorted populations were subsequently analyzed using

345 immunohistochemistry and qRT-PCR for expression of CD45 and EpCAM for each of the 
346 populations (Figure 3). To rule out epitope sharing as the cause of the bone marrow-derived DP

347 population, we determined the relative level of mRNA expression of both CD45 and EpCAM

348 compared to the housekeeping gene, 18s rRNA. All RNA samples isolated for these

349 experiments were diluted to $5 \mathrm{ng} / \mu \mathrm{L}$, to exclude differences in RNA amounts and normalized to

350 18s rRNA. The CD45+EpCAM+ population was shown to express both CD45 and EpCAM

351 mRNA using qRT-PCR (Figure 3B-C). Figure 3 panel B shows the relative CD45 expression in

352 the CD45+EpCAM+ population when compared with the CD45+EpCAM- positive control

353 population. As expected, the CD45+ population has a high level of CD45 mRNA expression.

354 The DP population clearly expresses CD45 mRNA. However, it has a $~ 83$-fold lower

355 expression level than the CD45+ EpCAM- control population. Panel C shows the relative

356 EpCAM expression in comparison to the CD45+EpCAM-, negative control population. The

357 CD45 only population has a 100-fold lower EpCAM mRNA expression than the DP

358 population.

359 The sorted DP and CD45 only populations in the BM were also analyzed using

360 immunohistochemistry for surface protein expression of CD45, EpCAM, and a mixture of

361 CD11b, CD11c, CD19 for each of the populations (Figure 3D-M). CD11b, CD11c, and CD19

362 combined staining was done to rule out $\mathrm{FC}_{\mathrm{C}}$ receptor binding to the CD45 and EpCAM

363 antibodies leading to a false-positive result. The control CD45+EpCAM- population, only

364 exhibited staining with the anti-CD45 antibody (3D-H). In contrast, the CD45+EpCAM+ cells

365 were stained with both the anti-CD45 (3J) and anti-EpCAM antibodies (3L) while no staining

366 with the CD11b, CD11c, CD19 cocktail (3K) was observed. The inset shows a higher

367 magnification image of the DP cells (outlined by the box) for every staining panel and

368 demonstrates that the DP cells represent single cells expressing both CD45 and EpCAM. 
369 Together these IHC results of sorted cells confirmed that single CD45+EpCAM+ cells co-

370 express both the CD45 and EpCAM proteins on their cell surface.

371

372 Collectively, these data show that there is a rare population of previously undescribed cells in

373 both adult thymus and the bone marrow that co-express the hematopoietic marker CD45 and the

374 definitive thymic epithelial marker EpCAM. Further, we have demonstrated that in both adult

375 bone marrow and thymus, single cells express both the CD45 and EpCAM surface proteins and

376 that highly purified sorted populations express mRNA for both genes confirming that this rare

377 and unique population exists. Absence of TNC complexes ensures that contrary to previous

378 reports when careful double discrimination is used, the DP cells are not TNCs, while the

379 absence of CD11c, CD11b, and CD19 surface protein expression allows exclusion of FC

380 receptor binding to antibodies or identification of Langerhans cells which are known to express

381 both CD11c and $\operatorname{EpCAM(48).~}$

382

$383 \mathrm{CD} 45+\mathrm{EpCAM}+$ cells are recruited to thymic stroma from the periphery and contribute to

384 EpCAM+CD45- TECs.

386 In order to understand if peripheral CD45+EpCAM+ cells can contribute to the non-

387 hematopoietic populations of the thymic stroma, E14.5 C57BL/6 fetal thymic lobes were

388 transplanted under the kidney capsule of Actin H2BGFP transgenic mice. The engrafted thymic

389 lobes were then harvested at different time points from 3 to 12 weeks following transplant and

390 analyzed for the presence of H2B-GFP-expressing cells that have migrated into and contributed

391 to the growing fetal thymi using both flow cytometry and immunohistochemistry. As a control, 
392 E14.5 C57BL/6 fetal thymic lobes were transplanted under the kidney capsule of C57B16 mice

393 and harvested after three weeks for flow cytometric analysis to set up negative gating for GFP

394 expression.

395

396 Figure 4A shows representative FACS analysis of dissociated transplanted thymic lobes

397 analyzed at different time points after transplant, Fig 4B shows the percentage of

398 CD45+EpCAM+GFP+ cells migrating into the C57BL/6 GFP-negative thymus, and 4C shows

399 the frequency of CD45-EpCAM+GFP+ TECs that are derived from peripheral sources. Bar

400 graphs of the mean percentages of peripheral CD45+EpCAM+GFP+ and CD45-EpCAM+GFP+

401 populations found in the transplanted thymi are shown in Figure 4D-E.

402

403 The flow-cytometric analysis revealed an influx of GFP+CD45+EpCAM+ cells into the

404 engrafted fetal thymic lobes. The presence of CD45-EpCAM+GFP+ TECs was detected at a

405 later time-point in the BL6 engrafts (Figure 4). An influx of the peripherally derived

$406 \mathrm{CD} 45+\mathrm{EpCAM}+\mathrm{GFP}+$ population was observed at every timepoint. The average frequency of

407 the CD45+EpCAM+GFP+ population remained almost constant at week three, $17.88 \%(+/-$

$4087.3 \%)$, and week six, $18 \%(+/ 7.9 \%)$. At week nine and week twelve, the average frequency of

409 the CD45+EpCAM+GFP+ population increased to $26.53 \%(+/-19 \%)$ and $27.3 \%(+/-13 \%)$,

410 respectively. An unpaired, non-parametric one-tailed T-test demonstrated that there was a

411 significant increase in the frequency of peripherally derived GFP+CD45+EpCAM+ cells within

412 the C57BL6 fetal thymi that were transplanted under C57BL6 kidney capsules when compared

413 with C57BL/6 control thymi transplanted under the kidney capsules of non-GFP expressing

414 mice. 
415 We compared each time point with the control. All the time-points analyzed, when C57BL/6

416 fetal lobes, where transplanted under the kidney capsule of ActinH2BGFP mice, showed a

417 statistically significant number of CD45+EpCAM+GFP+ cells had migrated to the transplanted

418 thymi.

419

420 Interestingly, a small but progressive increase in the frequency of CD45-EpCAM+GFP+ TECs

421 was also observed, suggesting that true EpCAM-expressing TECs were also derived from

422 peripheral sources. The average frequency of GFP expressing TECs increased from $2.6 \%(+/-$

$4232 \%)$ at week three to $4.4 \%(+/-2 \%)$ at week six. At week nine, the average frequency of GFP

424 expressing TECs was $6.34 \%(+/-4 \%)$ and increased to $7.4 \%(+/-4 \%)$ at week twelve. C57BL/6

425 fetal thymi transplanted under the C57BL/6 kidney capsule were used as a control to allow

426 gating on true GFP expressing cells in the engrafted thymus. When the frequency of

427 EpCAM+GFP+ cells in the engrafted thymi at each time points was compared to the control

428 (using a non-parametric, unpaired, one-tailed T-test), it showed significant numbers of

429 EpCAM+GFP+ true TECs appearing in the transplanted thymic lobes. These results confirm

430 that peripheral GFP+ CD45+EpCAM+ cells migrate into the thymus and that these peripheral

431 cells then give rise to GFP-expressing CD45-EpCAM+ thymic epithelial cells. This result is

432 unprecedented, as it suggests that contrary to previous models of thymic organogenesis, which

433 suggested that all EpCAM expressing TECs were derived from the third pharyngeal pouch

434 endoderm and its derivatives, that in fact up to $7 \%$ of EpCAM only TECs may be derived from 435 peripheral sources that migrate into the thymus. 
437 Peripheral cells contribute to the pan-keratin and FoxN1 expressing epithelial cells in the 438 thymic stroma:

440 The engrafted thymi were also analyzed histologically in parallel experiments at the previously

441 indicated time points. Figure 5 shows the staining pattern of PanK (an epithelial cell marker)

442 and FoxN1 (a definitive thymic epithelial cell marker) in the transplanted thymic tissues at

443 different time points following transplant. These histological results showed the presence of a

444 PanK+ FoxN1+ H2B-GFP+ and PanK+FoxN1- H2BGFP population in the engrafted fetal

445 thymus at week three and week six and PanK+FoxN1+H2B-GFP+ cells appeared in higher

446 number at later time points (week nine and week twelve) shown in figure 5. Expression of PanK

447 on a GFP+ cell in the transplanted thymi indicates that peripheral cells can migrate into the

448 thymus and contribute to true TECs. At earlier time points, only PanK+FoxN1-GFP+ cells were

449 observed, while at later time points, PanK+FoxN1+GFP+ cells were observed. This sequential

450 emergence of two different populations of PanK+GFP+ cells that were initially FoxN1-

451 followed by FoxN1+ cells with progressive age, indicates that first peripheral cells are recruited

452 to the thymus and differentiate into only PanK expressing TEC, and at a later time point more

453 mature PanK+FoxN1-expressing cells emerge.

455 CD45+EpCAM+ cells can contribute to the thymic stroma in vivo.

456 To understand the differential potential of the CD45+EpCAM+ population in vivo, we used

457 reaggregate thymic organ culture (RTOC). GFP+CD45+EpCAM+cells were sorted to high

458 purity (greater than 95\%) from Actin H2BGFP mouse bone marrow or thymus and reaggregated

459 with dissociated fetal thymic cells (E14.5) derived from GFP- C57BL6 mice. Reaggregates 
were cultured for $48 \mathrm{hrs}$ on polycarbonate filters supported by trans-well plates and then engrafted under the kidney capsule of athymic nude mice. The resulting ectopic thymic graft

462 incorporated the GFP+CD45+EpCAM+ population into the thymic stroma. At week three, we

463 observed PanK and FOXN1 expressing GFP+ cells in thymic stroma (Figure 6). RTOCs derived

464 from bone marrow had more GFP+ TECs, shown in figure 6A-D, whereas thymus-derived

465 RTOCs had a limited GFP+ TEC population (6 E-H). These results together showed that bone marrow and thymus derived CD45+ EpCAM+ cells could give rise to thymic epithelium and

467 prove that peripheral cells contribute to the TEC component of the thymic stroma.

469 FSP1 expressing fibroblast cells in the thymus are derived from peripheral cells:

470 During our FACS and IHC analysis, we observed that apart from the CD45+GFP+,

$471 \mathrm{CD} 45+\mathrm{EpCAM}+\mathrm{GFP}+$, and CD45-EpCAM+GFP+ populations, there were a significant

472 number of GFP+ cells that did not express either CD45 or EpCAM. In addition to TECs,

473 another non-hematopoietic contributor of the thymic stroma is fibroblasts. Recently, Fibroblast

474 Specific Protein-1 (FSP-1) expressing cells were shown to help to maintain medullary thymic

475 epithelial cells(51). Engrafted thymi were analyzed for FSP1 expression using IHC (Figure 7).

476 Figure 7A-P shows a representative figure of FSP1 staining in the transplanted thymi at

477 different time-points. The frequency of peripherally derived GFP+ cells found in the

478 transplanted fetal lobes that express FSP1 was calculated at the different time-points post-

479 transplant. At week three, the percentage of GFP+ cells expressing FSP1 was 35\% (+/- 8\%),

480 while at week six, it increased to 53 (+/-13). At week nine and week twelve, the percentage

481 increased to $71.2 \%(+/-8 \%)$ and $71.4 \%(+/-10 \%)$, respectively( Supplemental figure 2$)$. These

482 results suggest that FSP1-expressing cells in the thymic stroma are continuously derived from 
483 cells migrating into the thymus from peripheral sources rather than through the expansion of

484 stromal cells derived during embryonic development of the thymus.

486 CD45+EpCAM+ cells can contribute to the FSP1 expressing fibroblasts in the thymic stroma in 487 vivo.

489 We next wanted to investigate whether the BM and thymic derived CD45+EpCAM+ cells that 490 contribute to TECs might also contribute to the peripheral populations giving rise to FSP1-

491 expressing stromal components in the thymus. RTOCS composed of sorted

$492 \mathrm{CD} 45+\mathrm{EpCAM}+\mathrm{H} 2 \mathrm{BGFP}+\mathrm{BM}$ or thymic cells mixed with non-GFP expressing fetal thymic

493 cells were sectioned and stained with FSP1 to investigate if CD45+EpCAM+ cells can

494 contribute to the FSP1+ fibroblast population of the thymic stroma (Figure 8). We observed

495 FSP1 expressing H2B-GFP+ cells in the thymic stroma of RTOCs derived from both bone

496 marrow and thymic-derived CD45+EpCAM+ cells showing that CD45+EpCAM+ cells can also

497 give rise to FSP1 expressing fibroblasts.

499 Peripheral derived CD45+EpCAM+H2BGFP+ and CD45-EpCAM+H2BGFP+ cells are the 500 results of trans-differentiation and not cell fusion.

501 Previous studies reported that HSC cells could give rise to epithelial cells in different organs, 502 including the liver, lung, GI tract, and skin through trans-differentiation(35, 40). However, these 503 results were later disputed by Terada et al. and Wang et al., which suggested that BM-derived 504 cells do not transdifferentiate, but they spontaneously fuse with other cells and adopt their 505 characteristics $(43,44)$. The relatively high frequency of CD45+EpCAM+ cells observed in both 
506 the thymus and BM suggested that they were not derived from fusion. To determine if the

507 H2BGFP-expressing TECs we observed in transplant experiments were the result of cell fusion

508 events or true trans-differentiation, we transplanted E14.5 fetal thymuses derived from Actin

509 H2BGFP time-pregnant mice under the kidney capsule of Rosa26 mRFP mice. If actin-

$510 \mathrm{H} 2 \mathrm{BGFP}+$ EpCAM expressing CD45+EpCAM+ cells and the CD45-EpCAM+ true TECs

511 shown in Figure 4 are the result of cell fusion, then in this double transgenic model both GFP

512 and RFP should be expressed together on both the CD45+EpCAM+ cells and their resulting

513 TEC progeny. However, if the cells are genuinely derived from peripheral sources, then co-

514 expression of both RFP and GFP in the CD45+ EpCAM+ and EpCAM+ populations should not

515 be observed. We analyzed the transplanted thymi at two different time points 6 and 9 weeks

516 after transplant. Analysis of dissociated GFP-expressing fetal lobes using flow cytometry to

517 identify RFP and GFP 6 and 9 weeks after transplant under the kidney capsule of Rosa26 mRFP

518 mice revealed no co-expression of GFP and mRFP in either the CD45- EpCAM+, CD45+

519 EpCAM+ populations (Supplemental Figure 3.). mRFP+ peripheral cells were observed in all

520 the populations, including CD45-EpCAM+ TECs. The CD45+EpCAM+ cell population had

521 similar frequencies of peripheral mRFP+ cells at both time points; $68 \%(+/-15 \%)$ and $66 \%(+/-$

522 15), respectively. The CD45-EpCAM+ population also contained $\sim 1 \%$ RFP + cells that were

523 derived from peripheral mRFP cells migrating into the lobes. This set of results showed that

524 peripheral CD45+EpCAM+ cells that migrate to the thymus and contribute to the thymic stroma

525 are not the result of cell fusion and any H2BGFP+CD45+EpCAM+ cells observed as well as

526 their H2BGFP+ CD45-EpCAM+ TEC progeny represent peripheral cells migrating into the

527 thymus and contributing to stromal components. 
Discussion:

530 In this study, we describe a unique, rare population in the postnatal bone marrow, which can

531 migrate to the thymus and contribute to the thymic epithelial cell pool. This bone marrow-

532 derived population expresses both the definitive thymic epithelial cell marker EpCAM and pan-

533 hematopoietic cell marker CD45. This unique population was also identified in dissociated

534 thymic tissue as well, supporting the idea that it migrates from the bone marrow to the thymus.

535 Immunohistochemistry and qRT-PCR results performed with highly purified sorted populations

536 of these CD45+EpCAM+ cells derived from both the bone marrow and thymus showed that

537 these cells express both CD45 and EpCAM at both the mRNA and protein levels, confirming

538 that they are not thymic nurse cells as previously reported(47) or an artifact derived from

539 enzymatic dissociation of closely associated TECs and thymocytes (Figure 2 and Figure 3 ).

541 Several previous studies suggested that bone marrow-derived populations could contribute to

542 the epithelial cell populations of different organs, including lung, stomach, intestine, uterus(35,

$54338,40,41)$; however, these studies never identified a contribution to the thymus. In this study,

544 for the very first time, we are showing the presence of a potential thymic epithelial progenitor

545 cell population within the bone marrow. Analysis of C57BL6 fetal thymi, after being

546 transplanted under the kidney capsule of Actin H2BGFP mice, revealed that the

547 CD45+EpCAM+ population can migrate from the periphery to a growing fetal thymus and can

548 contribute to non-hematopoietic components of the thymic stroma (Figure 4). With progressive

549 time after transplant, the frequency of the CD45+EpCAM+GFP+ cell population increased and

550 was followed by the subsequent emergence of EpCAM+GFP+ TECs in the transplanted

551 thymus. 
552 IHC analysis of the same tissue showed the presence of Pan-keratin and GFP expressing cells in

553 the transplanted tissues at all time points (Figure 5). Interestingly, Pan-keratin+FoxN1+GFP+

554 cells also appear to emerge at later time points. Foxn 1 is a crucial transcription factor in TEC

555 development and thymic function, and it is a well-established marker for thymic epithelial cells.

556 The sequential expression of FoxN1 leads us to believe that peripheral cells can migrate to the

557 growing thymus and can contribute to thymic stroma as PanK expressing thymic epithelial cells,

558 which later differentiate into more mature FoxN1 expressing TEC at later time points $(52,53)$.

560 The presence of relatively abundant GFP+ cells in grafted fetal thymic lobes that did not bind

561 the pan-keratin antibody, CD45, or Foxn1 led us to analyze the engrafted lobes for the presence

562 of other non-hematopoietic stromal components including fibroblasts using FSP1 (a fibroblast

563 marker). Recently it has been shown that FSP1 expressing cells are essential for the

564 maintenance of medullary thymic epithelial cells(51). FSP1 staining of the transplanted tissue

565 revealed the presence of a GFP expressing peripherally derived FSP1+ cell population (Figure

566 7). We also observed with progressive time that the number of peripherally derived

567 GFP+FSP1+ cells increased (Supplemental Figure 2).

568 These results together showed that a peripheral CD45+EpCAM+ cell population could migrate

569 to the thymus and contribute to the non-hematopoietic components of the thymic stroma. The

570 CD45+EpCAM+ population appears to contribute to thymic stroma in two different ways: 1) by

571 directly giving rise to EpCAM and keratin expressing epithelial cells and 2) giving rise to an

572 FSP1 expressing cell population, known to support the maintenance of the medullary thymic

573 epithelial cell population. Limitations in the availability of antibodies to sort FSP1-expressing

574 cells or co-localize both Keratin and FSP1 on the same populations post-RTOC precluded 
575 determining whether they represented the same population or unique lineages derived from the

576 CD45+EpCAM+ population. Future experiments will need to be performed to determine the

577 lineage relationship between FSP1+ GFP + peripheral cells and PanK+GFP+ peripherally

578 derived TECs.

579

580 Although several previous studies showed that bone-marrow-derived populations including

581 HSCs could contribute to tissue regeneration in epithelial organs, some of this work had been

582 refuted by subsequent studies which demonstrated that bone marrow-derived cells undergo

583 fusion allowing them to take on characteristics of the recipient organs cell phenotype, instead of

584 genuinely transdifferentiating(43). Our results obtained when ActinH2BGFP fetal thymus was

585 transplanted under the kidney capsule of mRFPRosa26 mice clearly showed that the

$586 \mathrm{CD} 45+\mathrm{EpCAM}+\mathrm{RFP}+$ cell population observed in the ActinH2BGFP thymus after transplant

587 is not the result of fusion (supplemental Figure 3) since no cells co-expressing RFP and GFP

588 were observed. The RFP+ CD45-EpCAM+ TECs observed in the transplanted thymi are

589 derived from peripheral RFP+ cells migrating into the GFP-expressing fetal lobe.

590

591 When highly purified CD45+EpCAM+ GFP+ cells from the bone-marrow were reaggregated

592 with non-GFP fetal thymic stroma, the GFP+ bone-marrow-derived cells gave rise to pan-

593 keratin and FoxN1-expressing true thymic epithelial cells(Figure 6A-D), as well as FSP1

594 expressing fibroblast cells(Figure 8A-C). Similar results were obtained with reaggregates made

595 using the thymus-derived CD45+EpCAM+ population (Figure 6E-H and Figure 8D-F) .

596 Together these results confirm that the CD45+EpCAM+ cell population from both bone marrow

597 and thymus can differentiate to Pan-Keratin expressing epithelial cells as well as FSP1 
598 expressing fibroblasts. Our results suggest that the thymus-derived CD45+EpCAM+ cells in

599 RTOCs exhibit less potential to differentiate into of Pan-keratin expressing TECs than BM-

600 derived CD45+EpCAM+ cells supported by the lower frequency of Pan-Keratin+ true TECs.

601 This result leads us to hypothesize that the BM-derived cells have more potential to differentiate

602 in Pan-Keratin expressing TECs, whereas the thymus-derived CD45+EpCAM+ population

603 might have lost some of its potentials or some of the cells may be committed to a different fate

604 other than Pan-Keratin expressing epithelial cells.

605

606 In summary, this study demonstrates that a bone-marrow-derived population expressing both

607 CD45 and EpCAM can migrate to the thymus and can give rise to two different non-

608 hematopoietic cell populations in thymic stroma including both FSP1-expressing fibroblasts and

609 keratin/FoxN1- expressing TECs. It is not clear whether the CD45+EpCAM+ cells are derived

610 from HSCs or represent a distinct lineage of BM cells. Future studies will also need to be

611 performed to determine the lineage relationships between the FSP1+ cells and the true keratin

612 expressing TECs derived from the CD45+EpCAM+ population. They could represent distinct

613 fates of the same population or steps in an undefined ontogeny. It is also unclear how

614 significant the CD45+EpCAM+ population and its progeny are to TEC homeostasis or how they

615 might contribute to self-tolerance or T cell selection. Previous work has suggested that the

616 MHC background of the thymic microenvironment determines the MHC restriction of

617 developing T cells after BMTs(54). If BM-derived cells are migrating into the thymus and

618 contributing to the maintenance of TEC subsets, then it is surprising that they do not influence

619 the MHC restriction of developing thymocytes. Further studies needed to be done to

620 understand the ontogeny of this population and its contribution to distinct TEC subsets. If 
621 migrating CD45+EpCAM+ cells only contribute to mTECs, then it is possible that they do not

622 influence MHC restriction but might contribute to self-tolerance through negative selection or

623 the development of Regulatory T-Cell (Tregs).

624

625 Despite the presence of a progenitor cell population in BM that can contribute to TECs, the

626 thymus still undergoes age-related involution. Understanding the lineage of the

$627 \mathrm{CD} 45+\mathrm{EpCAM}+\mathrm{BM}$ population as well as the mechanisms responsible for the migration of

628 this population to the thymus and how they contribute to the maintenance of TEC number and

629 organization will be critical in counteracting age-associated involution, particularly in cancer

630 patients, due to enhanced degeneration in response to therapy. This study addresses a

631 significant unmet clinical need for thymus reconstitution by defining the contribution of bone-

632 marrow-derived thymic epithelial progenitor cells (TEPC) to the maintenance of TEC

633 microenvironments in the postnatal thymus. It should provide an additional target for

634 overcoming thymic atrophy and hence, the development of more strategic therapies for

635 immunological-based diseases and cancer. 


\section{Figure Legends:}

639 Figure 1. An alternative hematopoietic cell depletion method reveals a previously unidentified

640 CD45+ EpCAM+ population in the thymus. FACS profiles show viable single cells gated on

641 CD11c-, CD11b-and CD19- (lineage negative) cells. A- Shows the presence of only $0.084 \%$ of

642 CD45+EpCAM+ cells present in dissociated thymic tissue when using sheep-anti-CD45

643 antibody followed by anti-sheep magnetic beads for depletion of hematopoietic cells. B- Shows

644 enrichment of the CD45+EpCAM+ population to $0.51 \%$ derived from dissociated thymic tissue

645 when a cocktail of sheep anti-Thy1.2, anti-CD11b and anti-CD19 antibodies followed by anti-

646 sheep magnetic beads were used in place of anti-CD45. All the experiments were done in three

647 replicates.

649 Figure 2. Single sorted thymic CD45+ EpCAM+ cells express both CD45 and EpCAM surface 650 proteins and their mRNA. CD45+ EpCAM+ cells were sorted to high purity and analyzed for

651 CD45 and EpCAM expression using immunofluorescence microscopy and qRT-PCR.

652 CD45+EpCAM-; and CD45-EpCAM+ cells were sorted as controls. A: FACS analysis

653 representation of gating strategy used for cell sort. B: Shows relative CD45 expression level in

654 the thymus derived CD45+EpCAM+ cells compared to the CD45+EpCAM- and CD45-

655 EpCAM+ control populations; C: Shows the relative EpCAM expression level in the thymus

656 derived CD45+EpCAM+ cells compared to the CD45+EpCAM- and CD45-EpCAM+ control

657 populations. D-H: DIC image, CD45 staining (blue), CD11b,CD11c,CD19 staining (green);

658 EpCAM staining (red) and Merged image respectively for CD45+EpCAM- control population ;

659 I-M: DIC image, CD45 staining(blue), CD11b,CD11c,CD19 staining (green); EpCAM staining 660 (red) and Merged image respectively for CD45-EpCAM+ control population; N-R: DIC image, CD45 staining (blue), CD11b,CD11c,CD19 staining (green); EpCAM staining 
661 (red) and Merged image respectively for the CD45+EpCAM+ experimental population.

662 CD45+EpCAM+ cells of interest are enlarged in the insets to show colocalization of both

663 proteins and represent the cells shown in the white boxes.

664

665 Figure 3. Single sorted bone marrow derived CD45+ EpCAM+ cells express both the CD45 and

666 EpCAM genes and surface proteins. CD45+ EpCAM+ cells were sorted to high purity and

667 analyzed for CD45 and EpCAM expression using immunofluorescence microscopy as well as

668 qRT-PCR. CD45+EpCAM- cells were also sorted as a control. A: FACS profile of the gating

669 strategy used for sorting. B: Shows the relative CD45 expression level in the sorted BM derived

670 CD45+EpCAM+ cells compared to the CD45+EpCAM- control population; C: Shows relative

671 EpCAM expression level in the sorted BM derived CD45+EpCAM+ cells compared to the

672 CD45+EpCAM- control population. D-H sorted CD45+EpCAM- controls (D) DIC image; (E)

673 CD45 staining (blue); (F) CD11b, CD11c, CD19 staining (green); (G) EpCAM staining (red);

674 (H) Merged. I-M shows immunohistochemistry results obtained with sorted CD45+EpCAM+

675 cells (I) DIC image; (J) CD45 staining (blue); (K) CD11b, CD11c, CD19 staining (green); (L)

676 EpCAM staining (red) and (M) Merged image. CD45+EpCAM+ cells of interest are enlarged in

677 the insets to show colocalization of both proteins and represent the cells shown in the white

678 boxes. All the experiments were done in three replicates.

680 Figure 4. A peripheral population can contribute both CD45+ EpCAM+ cells as well as CD45-

681 EpCAM+ TECs in the thymus. C57BL6 fetal thymi were transplanted under the kidney capsule

682 of Actin H2BGFP mice and analyzed for the presence of GFP-expressing cells at 4 time-points

6833 to 12 weeks after transplant using FACS. A: The FACS representation of the lineage depleted 
684 cells derived from dissociated engrafted thymi gated for CD45 and EpCAM. (B) The FACS

685 representation of the percentage GFP+ cells within the CD45+EpCAM+subset found in the

686 grafted thymus and (C) The FACS representation of the percentage GFP+ cells within the

687 CD45-EpCAM+ population at the different timepoints after transplant under the kidney capsule.

688 D. Bar graph showing the mean percentage of the GFP-expressing cells found within the

689 CD45+EpCAM+ cell population at different time-point (error bars represent SEM). E: Bar

690 graph showing the mean percentage of the GFP-expressing cells found within CD45-EpCAM+

691 TEC population at different time-points (error bars represent SEM). “*” indicates a statistically

692 significant difference between control and any time-point. P-values for each t-test analysis are

693 given with the bar graph. All the experiments were done in three-five replicates.

695 Figure 5: A peripheral population can migrate into the thymus and contribute to PanK and

696 FoxN1 expressing thymic epithelial cells. C57BL6 fetal thymi were transplanted under the

697 kidney capsule of Actin H2BGFP mice and analyzed for the presence of GFP-expressing

698 peripheral cells 3-12 weeks after transplant using IHC. A-D: Week 3 transplanted thymic tissue

699 sections showing expression of PanK (A), GFP+ peripheral cells (B), FoxN1 (C) and Merged

700 image (D). E-H: Week 6 transplanted thymic tissue section showing expression of PanK (E),

701 GFP+ peripheral cells $(\mathrm{F})$, FoxN1 (G) and Merged image (H). I-L: Week 9 transplanted thymic

702 tissue section showing expression of PanK (I), GFP+ peripheral cells (J), FoxN1 (K) and

703 Merged image (L). M-P: Week 12 transplanted thymic tissue section showing expression of

704 PanK (M), GFP+ peripheral cells (N), FoxN1 (O) and Merged image (P). GFP, Pank and

705 FoxN1 expressing cells of interest are enlarged in the insets to show colocalization of both

706 proteins and represent the cells shown in the white boxes. PanK+ FoxN1+ GFP+ cells and 
707 PanK+ FoxN1- GFP+ cells at all time points are indicated by white and red arrows respectively.

708 All the experiments were done in three-five replicates.

710 Figure 6. Reaggregate thymic organ cultures show that both bone marrow and thymus derived

711 CD45+ EpCAM+ cells can give rise to PanK+ FoxN1+ thymic epithelial cells. (A-D) Sections

712 of RTOC consisting of C57BL/6 fetal thymus mixed with sorted actin-H2BGFP-expressing

713 bone marrow-derived CD45+EpCAM+ cells. A representative RTOC section showing PanK

714 expression (A); FoxN1 expression (B) with GFP expressing cells derived from the

715 CD45+EpCAM+ cells sorted from adult GFP-expressing bone-marrow (C), (D) Merged image

716 of PanK, FoxN1 and GFP. (E-H) Sections of RTOC consisting of C57BL/6 fetal thymus mixed

717 with sorted actin-H2BGFP-expressing thymic derived CD45+EpCAM+ cells. A representative

718 RTOC section showing PanK expression (E); FoxN1 expression (F) and; GFP expressing cells

719 derived from actin H2B-GFP-expressing CD45+EpCAM+ cells sorted from adult thymus (G);

720 Merged image of PanK, FoxN1 and GFP (H). GFP, Pank and FOXN1 expressing cells of

721 interest are enlarged in the insets to show co-expression of both proteins and represent the cells

722 shown in the white boxes. All the experiments were done in three replicates.

724 Figure 7: Peripheral cells migrating into the thymus contribute to FSP1-expressing fibroblasts.

725 C57BL6 fetal thymi were transplanted under the kidney capsule of Actin H2BGFP mice and

726 analyzed 3-12 weeks after transplant using IHC. A-C: Week 3 transplanted thymic tissue

727 section showing the presence of GFP expressing peripheral cells (A), FSP1-expressing cells (B),

728 and Merged image for FSP1 and GFP expression (C). D-F: Week 6 transplanted thymic tissue

729 section shows the presence of GFP-expressing peripheral cells (D), staining pattern for FSP1 
730 (E), and Merged image for FSP1 and GFP expression (F). G-I: Week 9 transplanted thymic

731 tissue section shows presence of GFP expressing peripheral cell (G), staining pattern for FSP1

$732(\mathrm{H})$, and Merged image for FSP1 and GFP expression (I). J-L: Week 12 transplanted thymic

733 tissue section shows the presence of GFP-expressing peripheral cells (J), staining pattern for

734 FSP1(K), and Merged image for FSP1 and GFP expression (L). GFP, FSP1 expressing cells of

735 interest are enlarged in the insets to show co-expression of both proteins and represent the cells

736 shown in the white boxes. All the experiments were done in three- five replicates.

738 Figure 8. Reaggregate thymic organ cultures show that both bone marrow and thymus-derived

739 CD45+ EpCAM+ cells can give rise to FSP1+ thymic stromal cells;(A-C) RTOC consisting of

740 C57BL/6 fetal thymus mixed with bone marrow-derived actin H2B-GFP-expressing

741 CD45+EpCAM+ cells. A representative RTOC section showing FSP1 expression (A); with

742 GFP expressing cells derived from actin H2BGFP expressing CD45+EpCAM+ cells sorted

743 from adult bone-marrow (B); Merged image of FSP1 and GFP (C). (D-F) RTOC consisting of

744 C57BL/6 fetal thymus mixed with thymus-derived actin H2B-GFP-expressing CD45+EpCAM+

745 cells. A representative RTOC section showing FSP1 expression (D); with GFP expressing cells

746 derived from CD45+EpCAM+ cells sorted from adult bone-marrow (E); Merged image of FSP1

747 and GFP (F). GFP and FSP1 expressing cells of interest are enlarged in the insets to show co-

748 expression of both proteins and represent the cells shown in the white boxes. All the

749 experiments were done in three replicates.

750 
Literature Cited:

1. Zúñiga-Pflücker, J. C. 2004. T-cell development made simple. Nature Reviews Immunology 4: 67-72.

2. Anderson, G., and Y. Takahama. 2012. Thymic epithelial cells: working class heroes for $T$ cell development and repertoire selection. Trends in immunology 33: 256-263.

3. Anderson, G., N. C. Moore, J. J. Owen, and E. J. Jenkinson. 1996. Cellular interactions in thymocyte development. Annual review of immunology 14: 73-99.

9. Gray, D. H., N. Seach, T. Ueno, M. K. Milton, A. Liston, A. M. Lew, C. C. Goodnow, and R.

4. Takahama, Y. 2006. Journey through the thymus: stromal guides for T-cell development and selection. Nature Reviews Immunology 6: 127-135.

5. Anderson, G., E. J. Jenkinson, and H. R. Rodewald. 2009. A roadmap for thymic epithelial cell development. European journal of immunology 39: 1694-1699.

6. Chakravarti, B., and G. N. Abraham. 1999. Aging and T-cell-mediated immunity. Mechanisms of ageing and development 108: 183-206.

7. Cepeda, S., and A. V. Griffith. 2018. Thymic stromal cells: Roles in atrophy and ageassociated dysfunction of the thymus. Experimental gerontology 105: 113-117.

8. Barbouti, A., K. Evangelou, I. S. Pateras, A. Papoudou-Bai, A. Patereli, K. Stefanaki, D. Rontogianni, D. Muñoz-Espín, P. Kanavaros, and V. G. Gorgoulis. 2019. In situ evidence of cellular senescence in Thymic Epithelial Cells (TECs) during human thymic involution. Mechanisms of ageing and development 177: 88-90. L. Boyd. 2006. Developmental kinetics, turnover, and stimulatory capacity of thymic

10. Lynch, H. E., G. L. Goldberg, A. Chidgey, M. R. Van den Brink, R. Boyd, and G. D. Sempowski. 2009. Thymic involution and immune reconstitution. Trends in immunology 30: 366-373.

11. Rodewald, H.-R. 2008. Thymus organogenesis. Annu. Rev. Immunol. 26: 355-388.

12. Abramson, J., and G. Anderson. 2017. Thymic epithelial cells. Annual Review of Immunology 35: 85-118.

13. Anderson, G., S. Baik, J. E. Cowan, A. M. Holland, N. I. McCarthy, K. Nakamura, S. M. Parnell, A. J. White, P. J. Lane, and E. J. Jenkinson. 2013. Mechanisms of thymus medulla development and function. In Thymic Development and Selection of T Lymphocytes. Springer. 19-47.

14. Lind, E. F., S. E. Prockop, H. E. Porritt, and H. T. Petrie. 2001. Mapping precursor movement through the postnatal thymus reveals specific microenvironments supporting defined stages of early lymphoid development. Journal of experimental medicine 194: 127-134.

15. Blanpain, C., V. Horsley, and E. Fuchs. 2007. Epithelial stem cells: turning over new leaves. Cell 128: 445-458.

16. Bleul, C. C., T. Corbeaux, A. Reuter, P. Fisch, J. S. Mönting, and T. Boehm. 2006. Formation of a functional thymus initiated by a postnatal epithelial progenitor cell. Nature 441: 992-996. 
17. Rossi, S. W., A. P. Chidgey, S. M. Parnell, W. E. Jenkinson, H. S. Scott, R. L. Boyd, E. J. Jenkinson, and G. Anderson. 2007. Redefining epithelial progenitor potential in the developing thymus. European journal of immunology 37: 2411-2418.

18. Rossi, S. W., W. E. Jenkinson, G. Anderson, and E. J. Jenkinson. 2006. Clonal analysis reveals a common progenitor for thymic cortical and medullary epithelium. Nature 441:

19. Baik, S., E. J. Jenkinson, P. J. Lane, G. Anderson, and W. E. Jenkinson. 2013. Generation of both cortical and Aire+ medullary thymic epithelial compartments from CD205+ progenitors. European journal of immunology 43: 589-594.

20. Hamazaki, Y., H. Fujita, T. Kobayashi, Y. Choi, H. S. Scott, M. Matsumoto, and N. Minato. 2007. Medullary thymic epithelial cells expressing Aire represent a unique lineage derived from cells expressing claudin. Nature immunology 8: 304.

21. Rodewald, H.-R., S. Paul, C. Haller, H. Bluethmann, and C. Blum. 2001. Thymus medulla consisting of epithelial islets each derived from a single progenitor. Nature 414: 763768.

22. Shakib, S., G. E. Desanti, W. E. Jenkinson, S. M. Parnell, E. J. Jenkinson, and G. Anderson. 2009. Checkpoints in the development of thymic cortical epithelial cells. The Journal of Immunology 182: 130-137.

23. Ulyanchenko, S., K. E. O’Neill, T. Medley, A. M. Farley, H. J. Vaidya, A. M. Cook, N. F. Blair, and C. C. Blackburn. 2016. Identification of a bipotent epithelial progenitor population in the adult thymus. Cell reports 14: 2819-2832.

24. Ucar, A., O. Ucar, P. Klug, S. Matt, F. Brunk, T. G. Hofmann, and B. Kyewski. 2014. Adult thymus contains FoxN1- epithelial stem cells that are bipotent for medullary and cortical thymic epithelial lineages. Immunity 41: 257-269.

25. Peterson, P., and M. Laan. 2013. Bipotency of thymic epithelial progenitors comes in sequence. European journal of immunology 43: 580-583.

26. Mayer, C. E., S. Žuklys, S. Zhanybekova, I. Ohigashi, H. Y. Teh, S. N. Sansom, N. ShikamaDorn, K. Hafen, I. C. Macaulay, and M. E. Deadman. 2016. Dynamic spatio-temporal contribution of single $\beta 5 t+$ cortical epithelial precursors to the thymus medulla. European journal of immunology.

27. Ohigashi, I., S. Zuklys, M. Sakata, C. E. Mayer, S. Zhanybekova, S. Murata, K. Tanaka, G. A. Holländer, and Y. Takahama. 2013. Aire-expressing thymic medullary epithelial cells originate from $\beta 5$ t-expressing progenitor cells. Proceedings of the National Academy of Sciences 110: 9885-9890.

28. Blau, H. M., T. Brazelton, and J. Weimann. 2001. The evolving concept of a stem cell: entity or function? Cell 105: 829-841.

29. Gordon, J., and N. R. Manley. 2011. Mechanisms of thymus organogenesis and morphogenesis. Development 138: 3865-3878.

30. Gray, D., A. Chidgey, and R. Boyd. 2002. Analysis of thymic stromal cell populations using flow cytometry. Journal of immunological methods 260: 15-28.

31. Sekai, M., Y. Hamazaki, and N. Minato. 2014. Medullary thymic epithelial stem cells maintain a functional thymus to ensure lifelong central T cell tolerance. Immunity 41: 753-761. 
32. Wong, K., N. L. Lister, M. Barsanti, J. M. Lim, M. V. Hammett, D. M. Khong, C. Siatskas, D. H. Gray, R. L. Boyd, and A. P. Chidgey. 2014. Multilineage potential and self-renewal define an epithelial progenitor cell population in the adult thymus. Cell reports 8: 11981209.

33. Osada, M., V. J. Singh, K. Wu, D. B. Sant'Angelo, and M. Pezzano. 2013. Label retention identifies a multipotent mesenchymal stem cell-like population in the postnatal thymus. PloS one 8: e83024.

34. Dumont-Lagacé, M., H. Gerbe, T. Daouda, J.-P. Laverdure, S. Brochu, S. Lemieux, É. Gagnon, and C. Perreault. 2017. Detection of quiescent radioresistant epithelial progenitors in the adult thymus. Frontiers in immunology 8: 1717.

35. Krause, D. S., N. D. Theise, M. I. Collector, O. Henegariu, S. Hwang, R. Gardner, S. Neutzel, and S. J. Sharkis. 2001. Multi-organ, multi-lineage engraftment by a single bone marrow-derived stem cell. Cell 105: 369-377.

36. Bittner, R. E., C. Schöfer, K. Weipoltshammer, S. Ivanova, B. Streubel, E. Hauser, M. Freilinger, H. Höger, A. Elbe-Bürger, and F. Wachtler. 1999. Recruitment of bonemarrow-derived cells by skeletal and cardiac muscle in adult dystrophic mdx mice. Anatomy and embryology 199: 391-396.

37. Mezey, É., K. J. Chandross, G. Harta, R. A. Maki, and S. R. McKercher. 2000. Turning blood into brain: cells bearing neuronal antigens generated in vivo from bone marrow. Science 290: 1779-1782.

38. Lagasse, E., H. Connors, M. Al-Dhalimy, M. Reitsma, M. Dohse, L. Osborne, X. Wang, M. Finegold, I. L. Weissman, and M. Grompe. 2000. Purified hematopoietic stem cells can differentiate into hepatocytes in vivo. Nature medicine 6: 1229.

39. Wong, A. P., A. E. Dutly, A. Sacher, H. Lee, D. M. Hwang, M. Liu, S. Keshavjee, J. Hu, and T. K. Waddell. 2007. Targeted cell replacement with bone marrow cells for airway epithelial regeneration. American journal of physiology-lung cellular and molecular physiology 293: L740-L752.

40. Wong, A. P., A. Keating, W.-Y. Lu, P. Duchesneau, X. Wang, A. Sacher, J. Hu, and T. K. Waddell. 2009. Identification of a bone marrow-derived epithelial-like population capable of repopulating injured mouse airway epithelium. The Journal of clinical investigation 119: 336.

41. Theise, N. D., S. Badve, R. Saxena, O. Henegariu, S. Sell, J. M. Crawford, and D. S. Krause. 2000. Derivation of hepatocytes from bone marrow cells in mice after radiation-induced myeloablation. Hepatology 31: 235-240.

42. Theise, N. D., M. Nimmakayalu, R. Gardner, P. B. Illei, G. Morgan, L. Teperman, O. Henegariu, and D. S. Krause. 2000. Liver from bone marrow in humans. Hepatology 32: 11-16.

43. Wang, X., H. Willenbring, Y. Akkari, Y. Torimaru, M. Foster, M. Al-Dhalimy, E. Lagasse, M. Finegold, S. Olson, and M. Grompe. 2003. Cell fusion is the principal source of bonemarrow-derived hepatocytes. Nature 422: 897.

44. Terada, N., T. Hamazaki, M. Oka, M. Hoki, D. M. Mastalerz, Y. Nakano, E. M. Meyer, L. Morel, B. E. Petersen, and E. W. Scott. 2002. Bone marrow cells adopt the phenotype of other cells by spontaneous cell fusion. Nature 416: 542. 
45. Borue, X., S. Lee, J. Grove, E. L. Herzog, R. Harris, T. Diflo, E. Glusac, K. Hyman, N. D. Theise, and D. S. Krause. 2004. Bone marrow-derived cells contribute to epithelial

engraftment during wound healing. The American journal of pathology 165: 1767-1772.

46. Amend, S. R., K. C. Valkenburg, and K. J. Pienta. 2016. Murine hind limb long bone

dissection and bone marrow isolation. JoVE (Journal of Visualized Experiments): e53936.

47. Nakagawa, Y., I. Ohigashi, T. Nitta, M. Sakata, K. Tanaka, S. Murata, O. Kanagawa, and Y. Takahama. 2012. Thymic nurse cells provide microenvironment for secondary T cell receptor alpha rearrangement in cortical thymocytes. Proc Natl Acad Sci U S A 109: 20572-20577.

48. Ouchi, T., G. Nakato, and M. C. Udey. 2016. EpCAM expressed by murine epidermal Langerhans cells modulates immunization to an epicutaneously applied protein antigen. Journal of Investigative Dermatology 136: 1627-1635.

49. Gaiser, M. R., T. Lämmermann, X. Feng, B. Z. Igyarto, D. H. Kaplan, L. Tessarollo, R. N. Germain, and M. C. Udey. 2012. Cancer-associated epithelial cell adhesion molecule (EpCAM; CD326) enables epidermal Langerhans cell motility and migration in vivo. Proceedings of the National Academy of Sciences 109: E889-E897.

50. Seré, K., J.-H. Baek, J. Ober-Blöbaum, G. Müller-Newen, F. Tacke, Y. Yokota, M. Zenke, and T. Hieronymus. 2012. Two distinct types of Langerhans cells populate the skin during steady state and inflammation. Immunity 37: 905-916.

51. Sun, L., C. Sun, Z. Liang, H. Li, L. Chen, H. Luo, H. Zhang, P. Ding, X. Sun, and Z. Qin. 2015. 909 
bioRxiv preprint doi: https://doi.org/10.1101/2020.09.29.319426; this version posted October 1, 2020. The copyright holder for this preprint (which was not certified by peer review) is the author/funder. All rights reserved. No reuse allowed without permission.

Figure 1
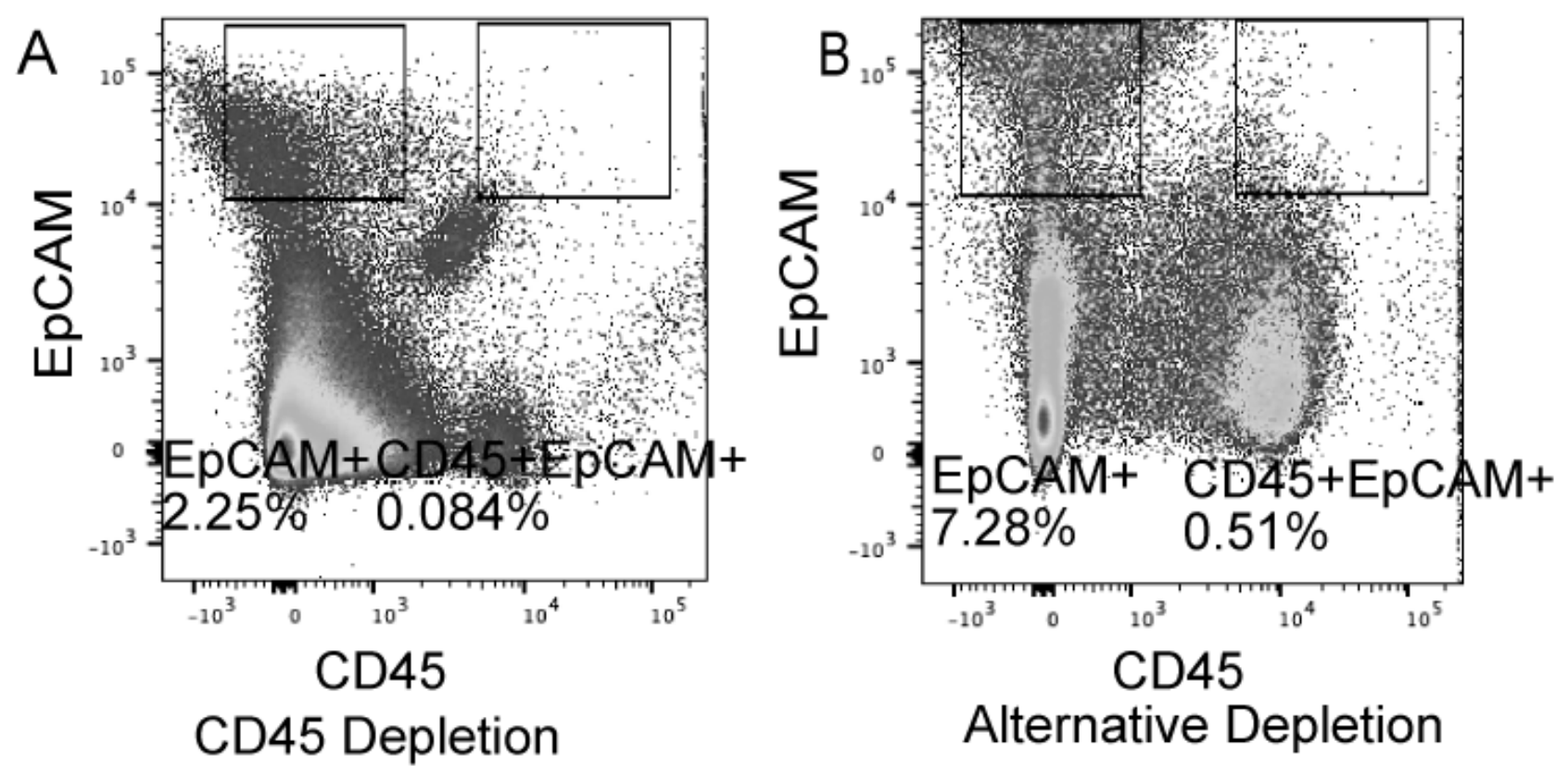

CD45

Alternative Depletion 
bioRxiv preprint doi: https://doi.org/10.1101/2020.09.29.319426; this version posted October 1, 2020. The copyright holder for this preprint Figure 2 (which was not certified by peer review) is the author/funder. All rights reserved. No reuse allowed without permission.
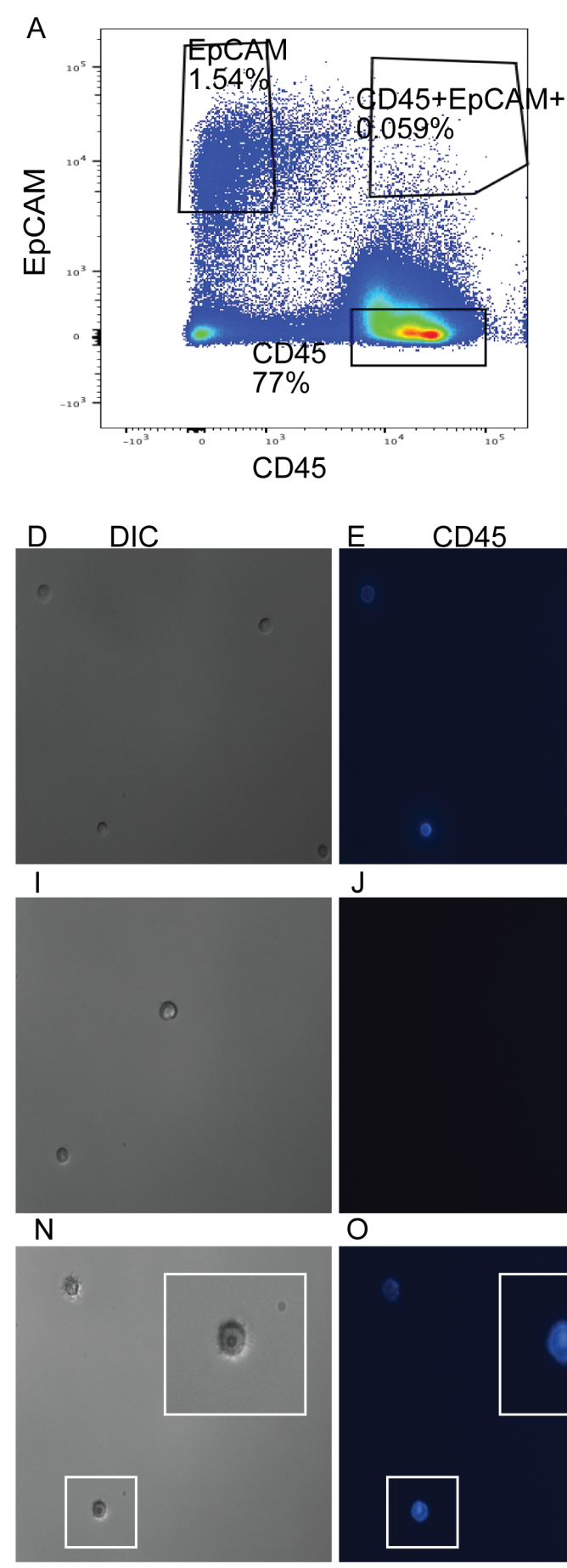

$\mathrm{J}$

O
B

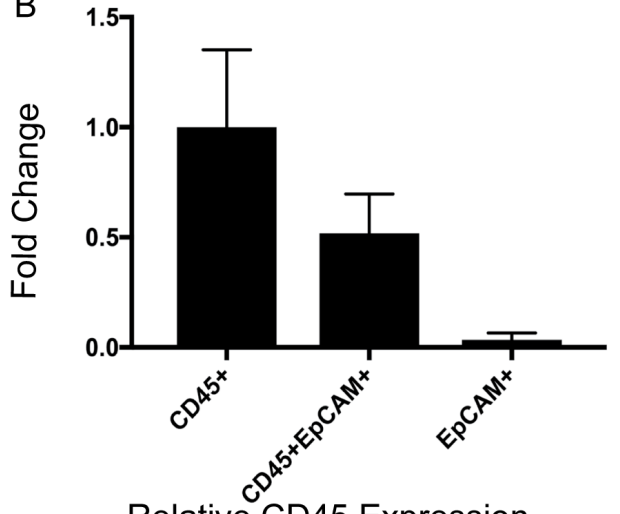

Relative CD45 Expression
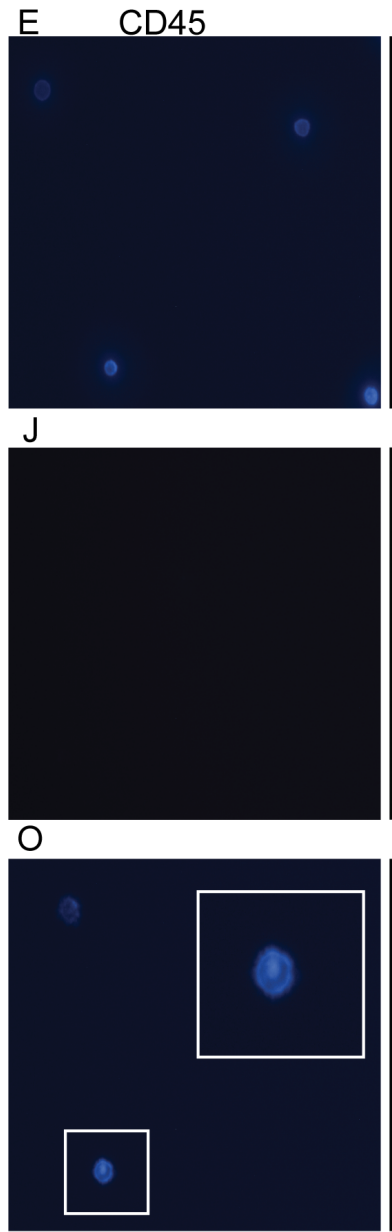

FCD11bCD11cCD19

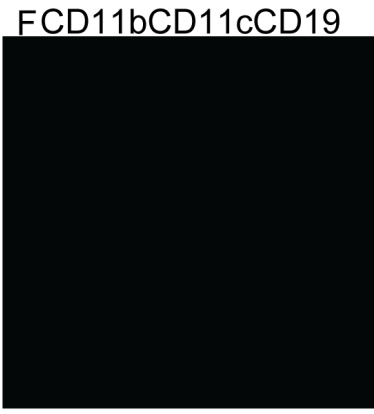

$\mathrm{K}$

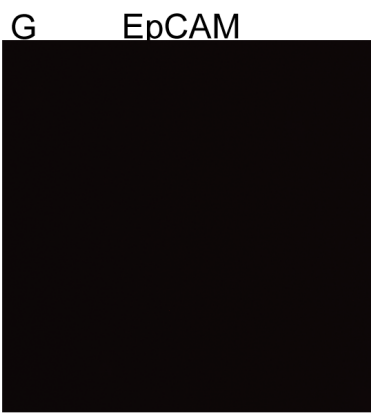

$\mathrm{L}$

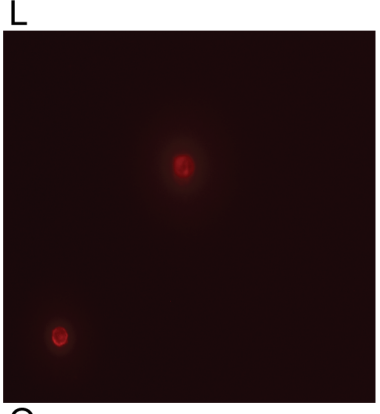

P

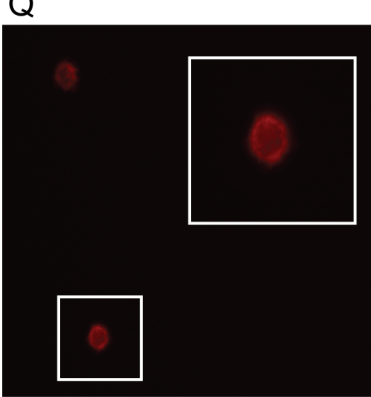

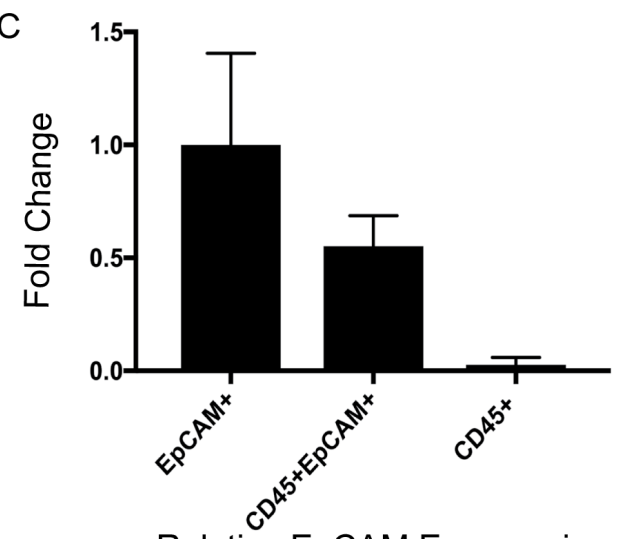

Relative EpCAM Exapression

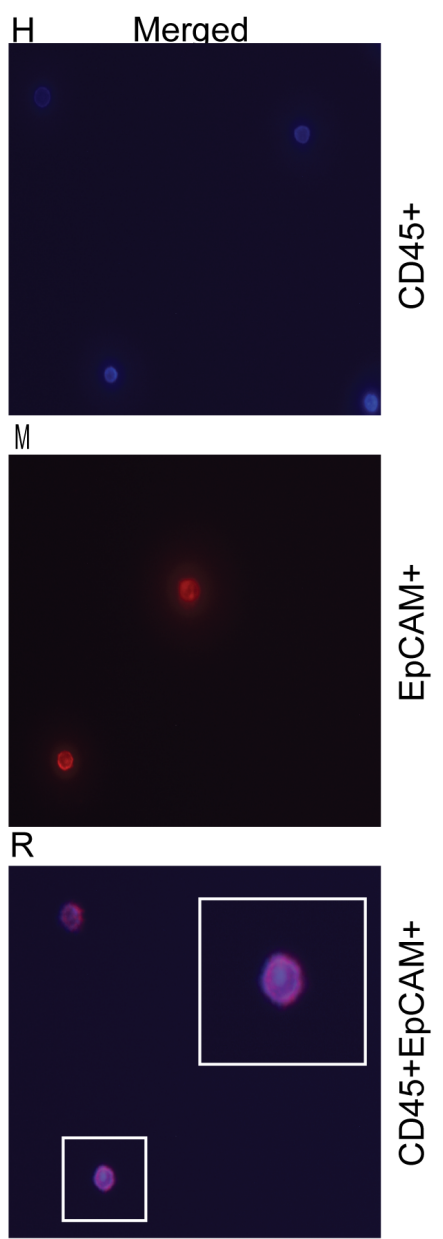


bioRxiv preprint doi: https://doi.org/10.1101/2020.09.29.319426; this version posted October 1, 2020. The copyright holder for this preprint Figure 3
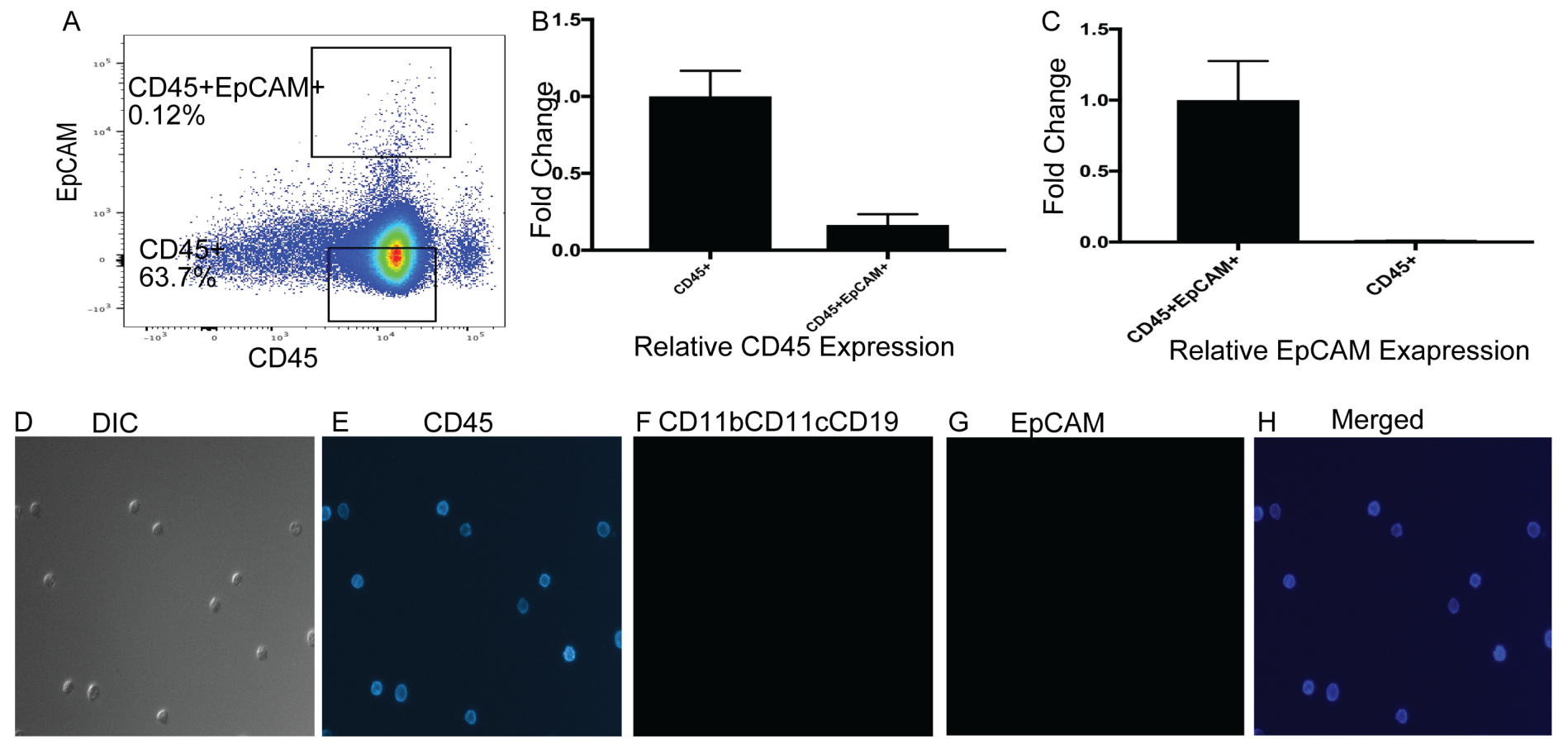

ปั
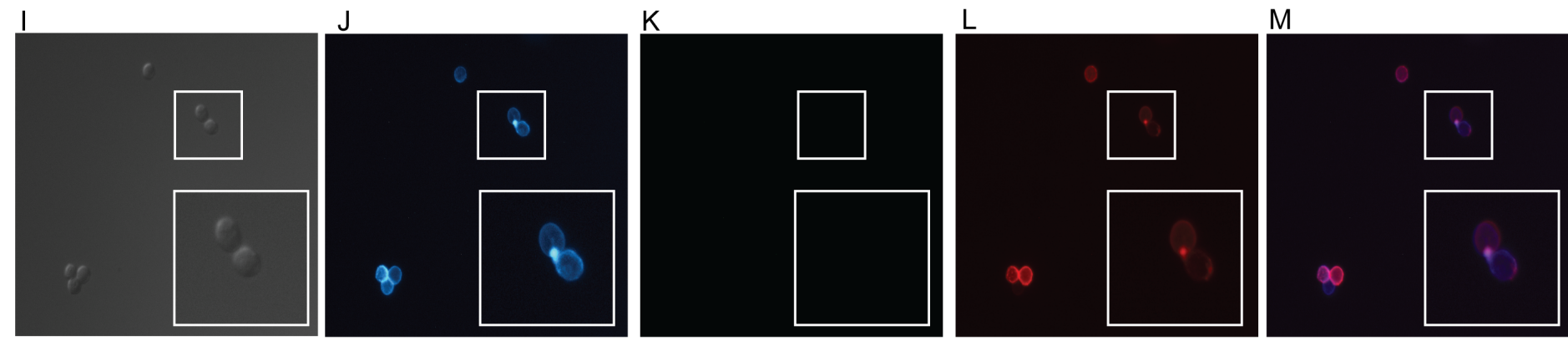

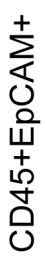


Figure 4

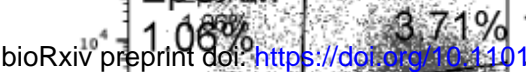

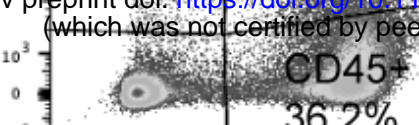
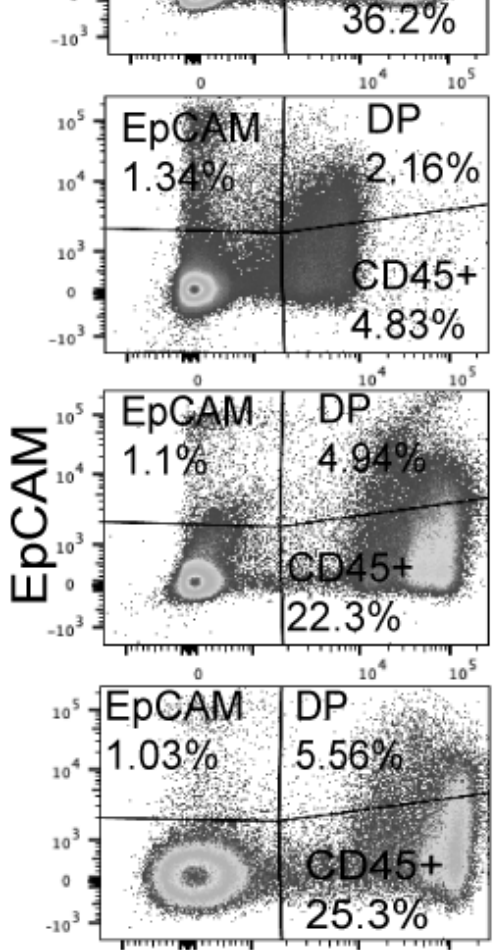

$10^{5} \sqrt[5]{\text { EpCAM DP }}$

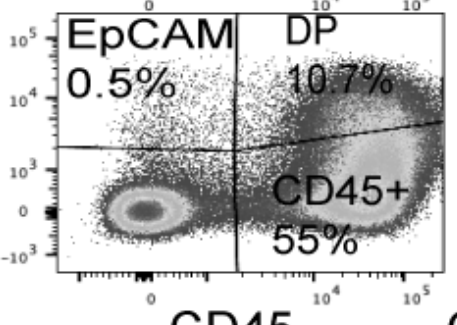

CD45
G GPP

C GFP

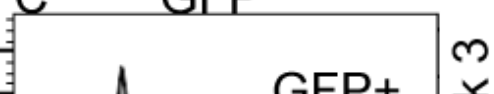

der for this preprint (iilssion.
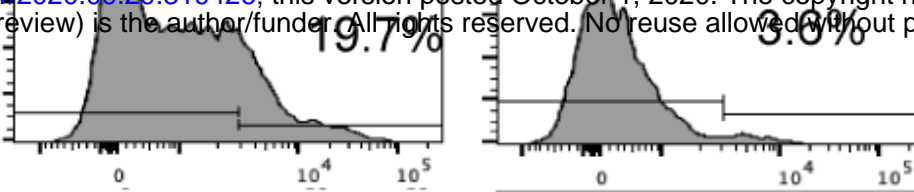

3
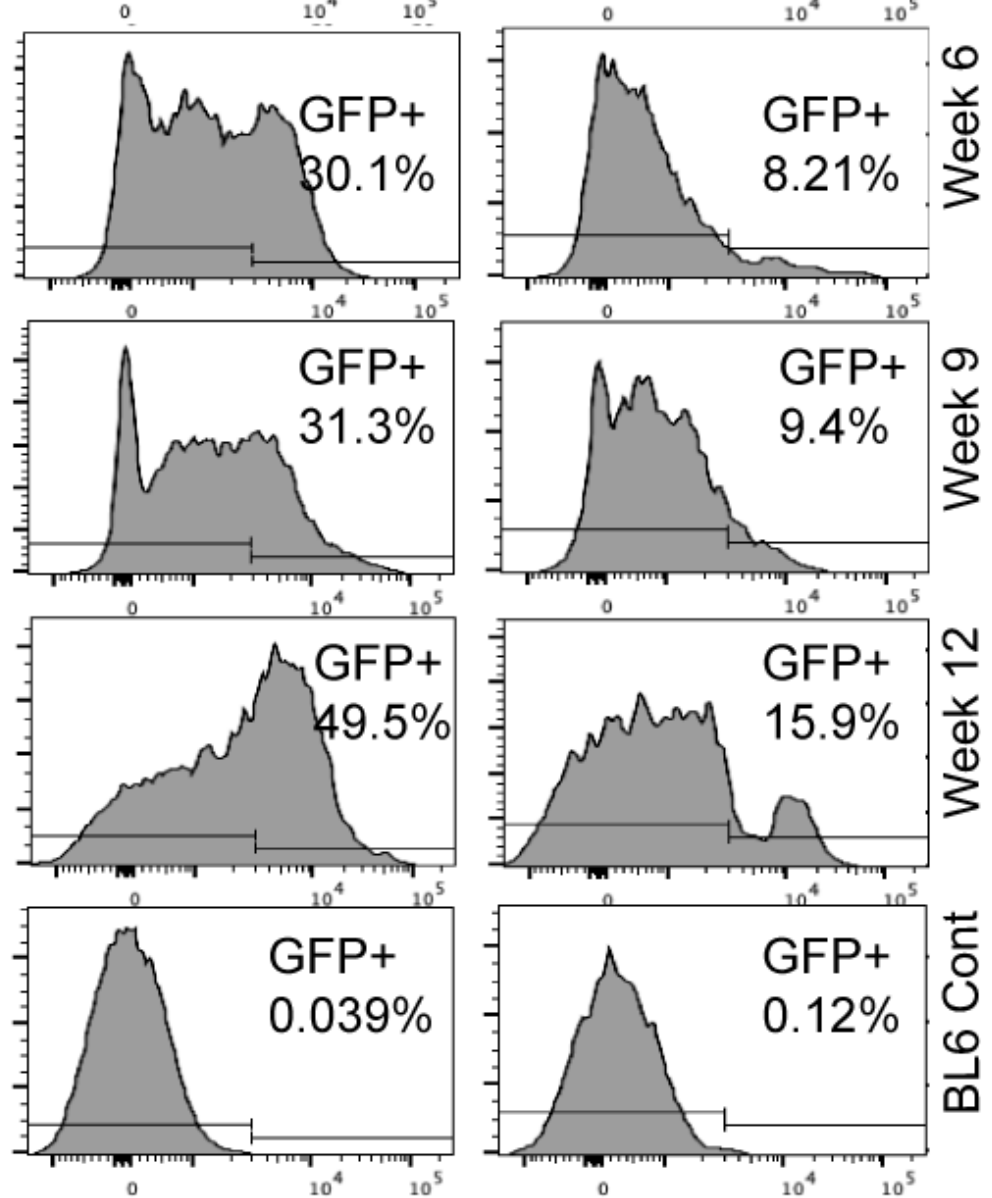

CD45+EpCAM+GFP+

$\mathrm{EpCAM}+\mathrm{GFP}+$
D $\mathrm{CD} 45+\mathrm{EpCAM+GFP+}$

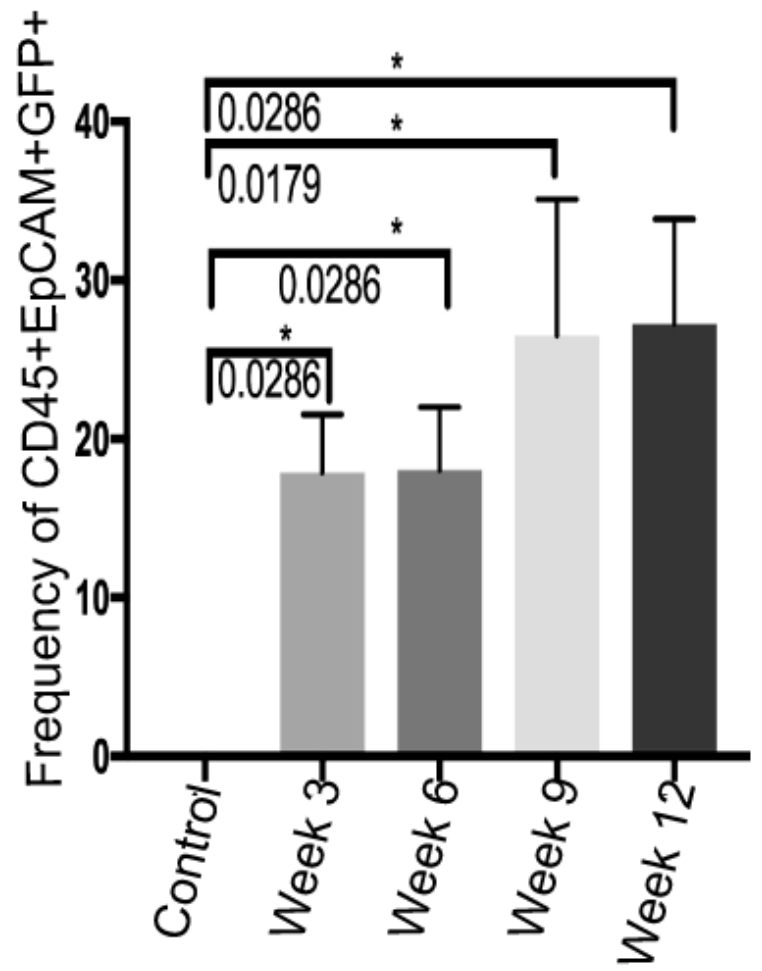

E EpCAM+GFP+

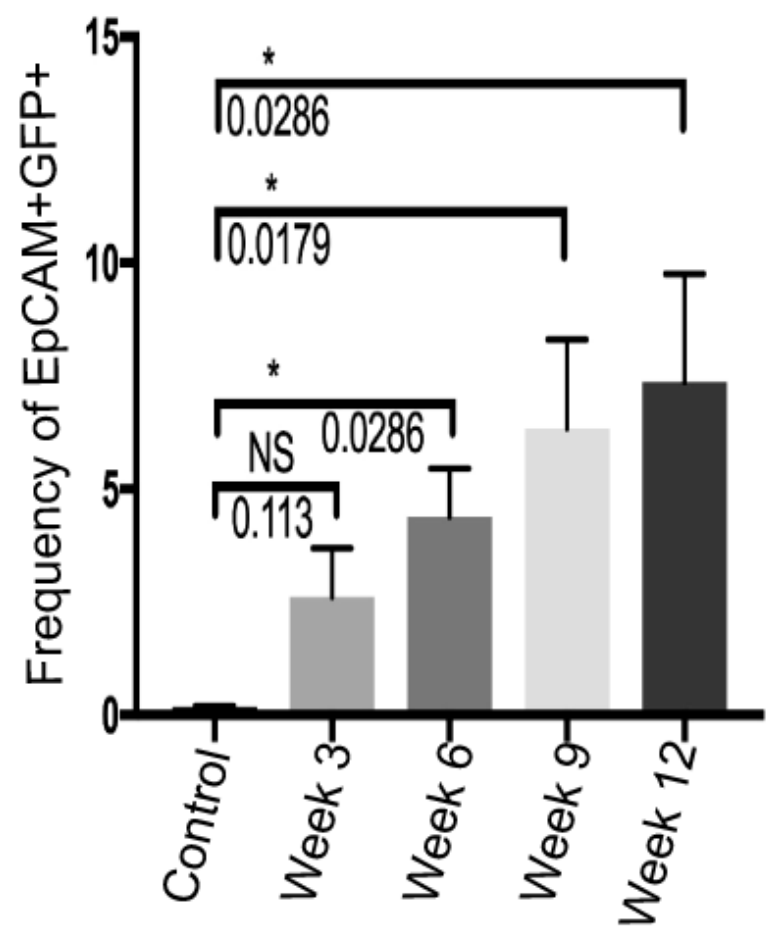


Figure 5
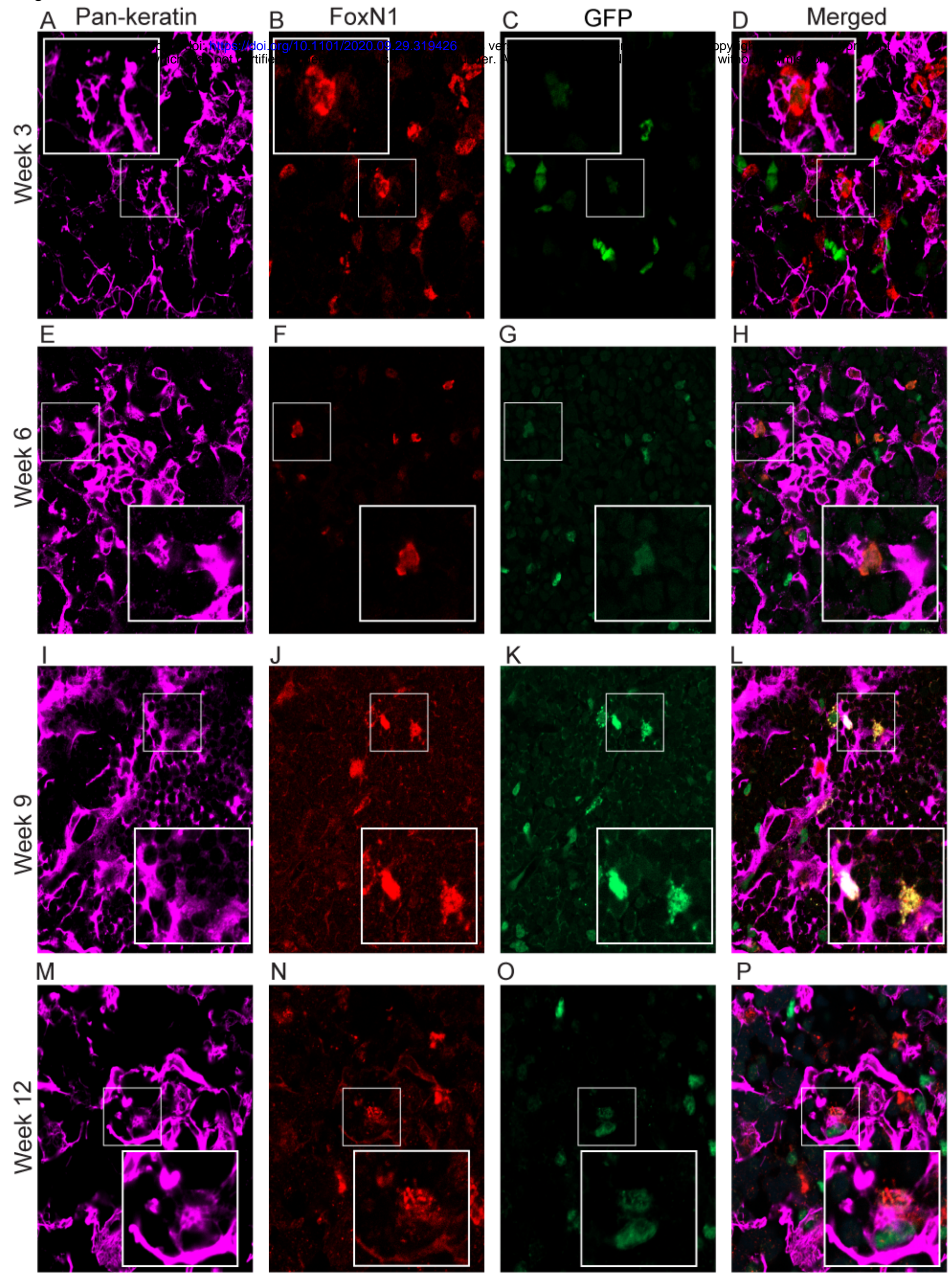
Figure 7
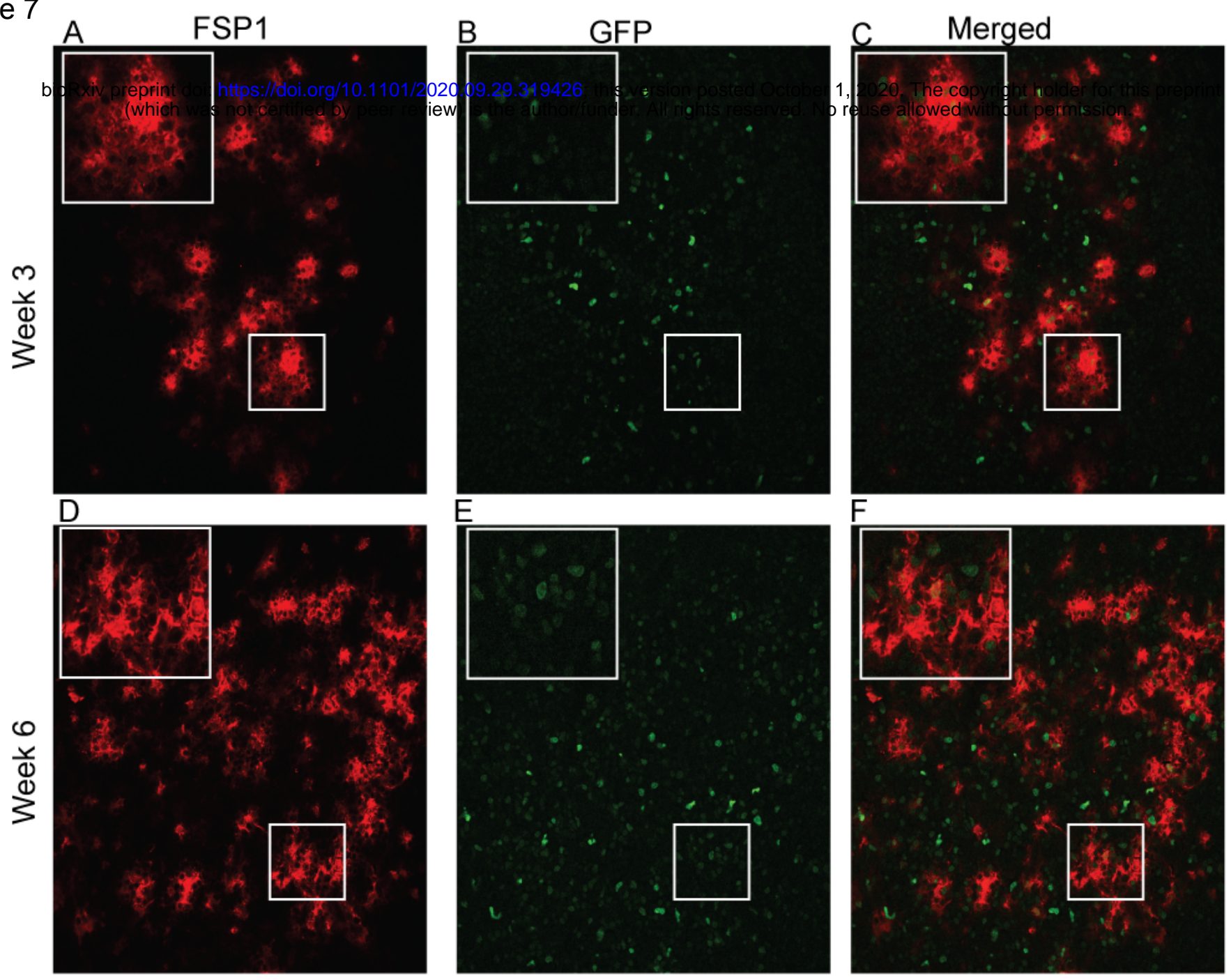

\section{G}

0
va
d
3

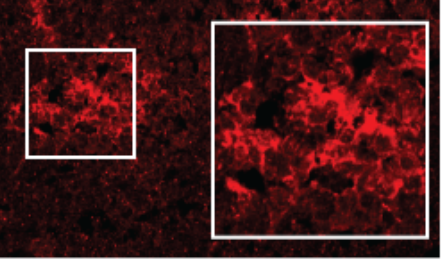

\section{$J$}

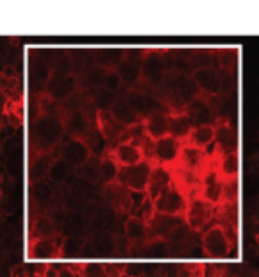

$\frac{\mathfrak{5}}{\stackrel{5}{0}}$

\section{$\mathrm{H}$}

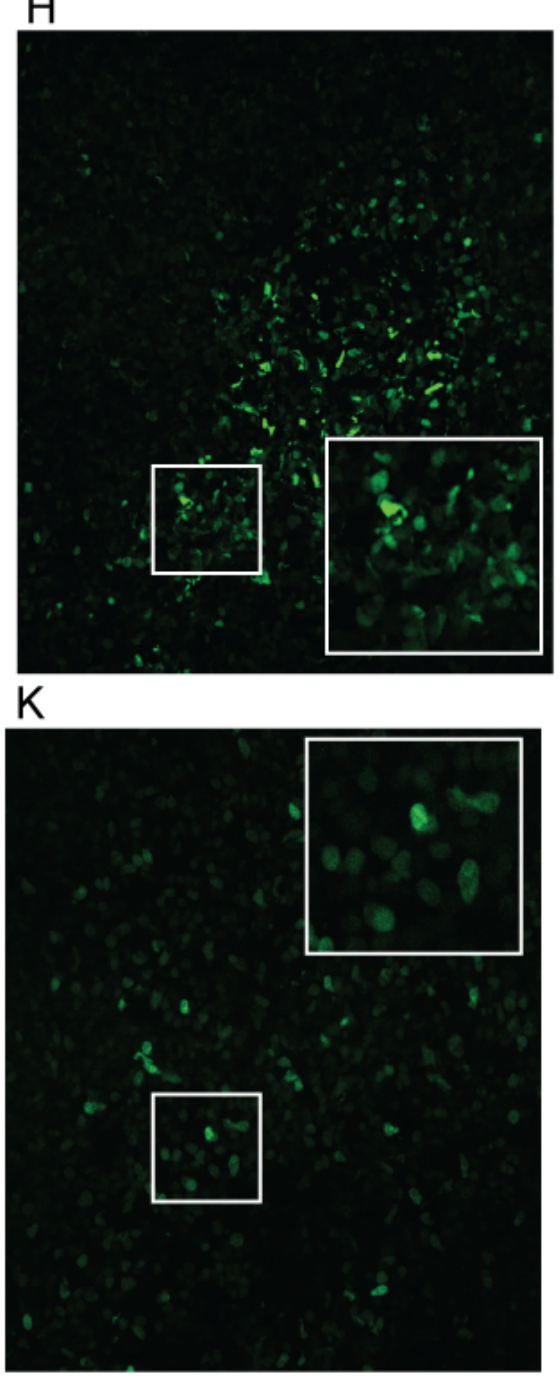

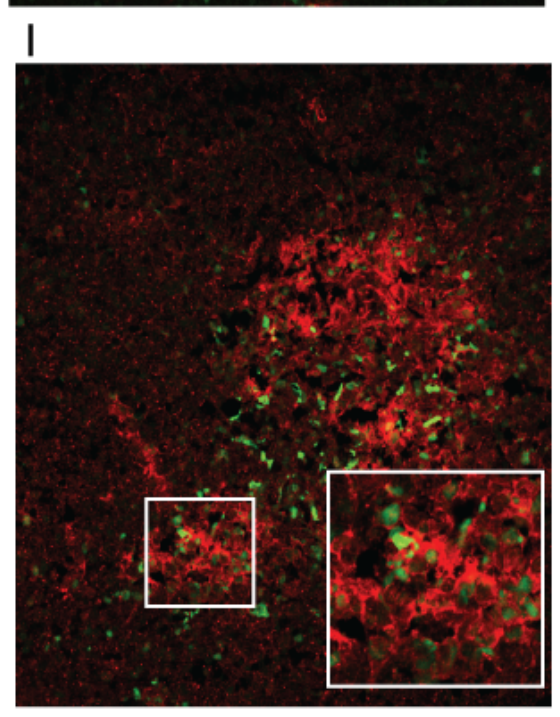

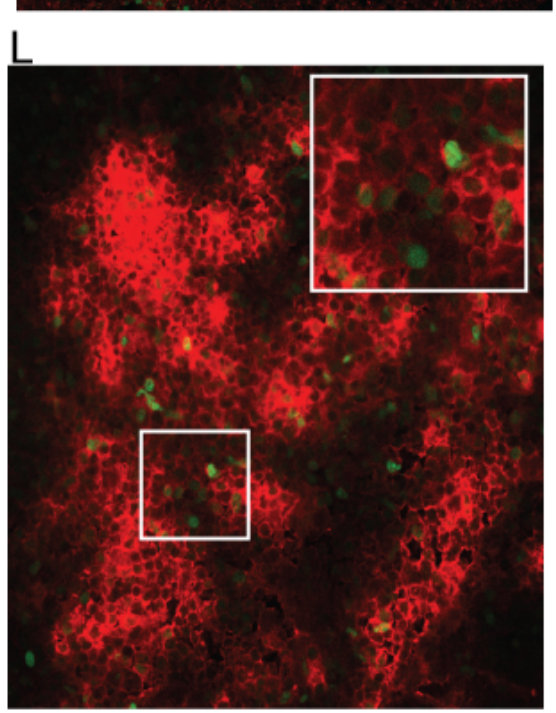


bioRxiv preprint doi: https://doi.org/10.1101/2020.09.29.319426; this version posted October 1, 2020. The copyright holder for this preprint Figure 8 (which was not certified by peer review) is the author/funder. All rights reserved. No reuse allowed without permission.

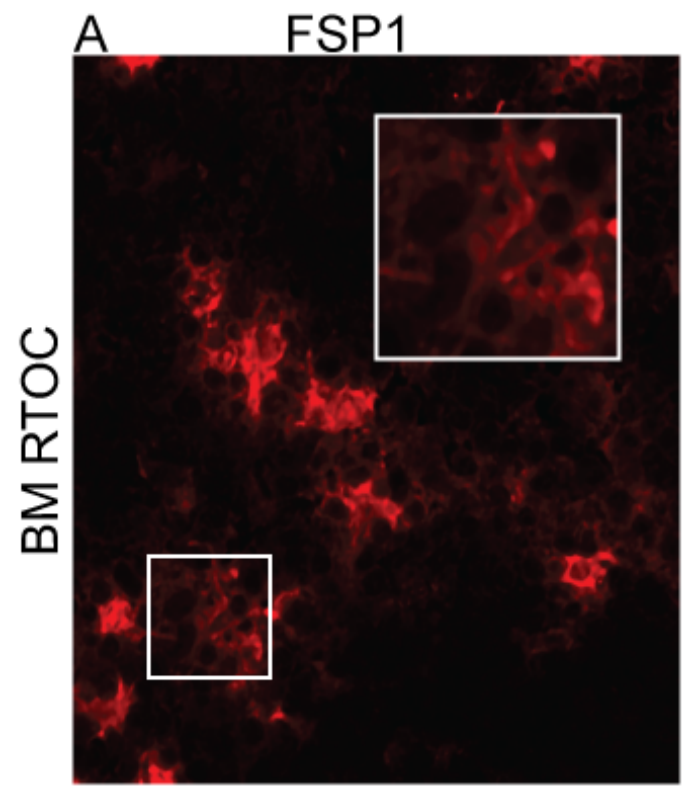

B

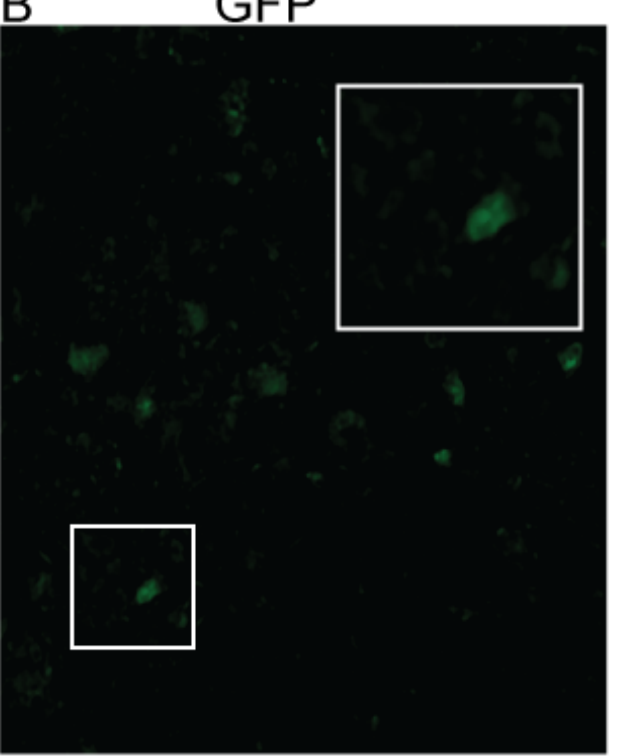

D

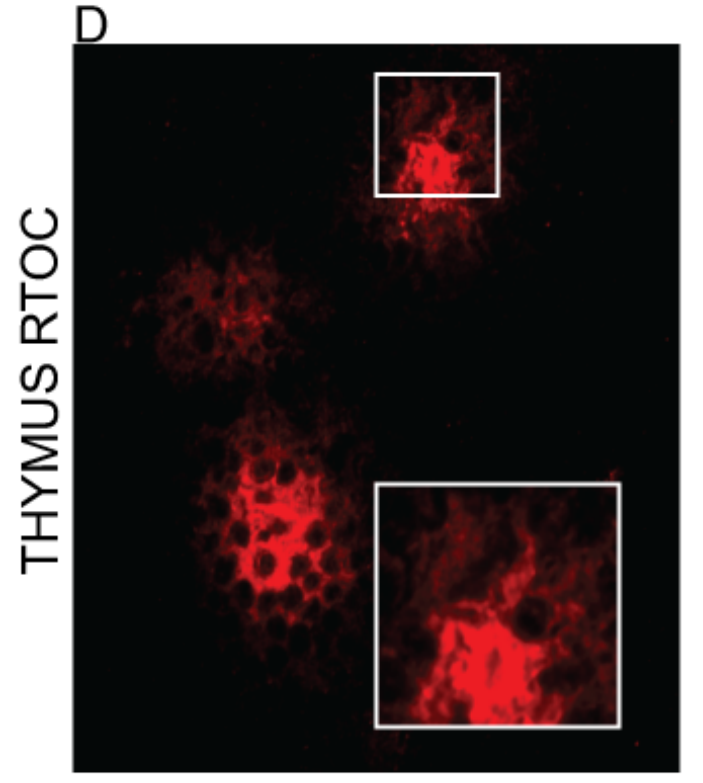

E

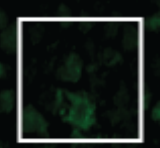

\section{Merged}
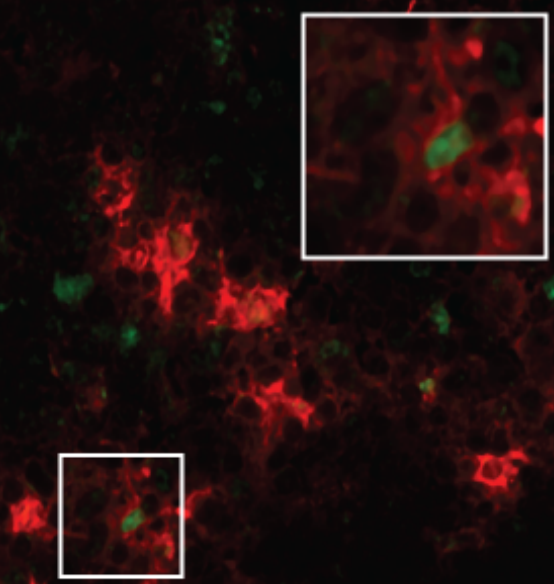

$\mathrm{F}$

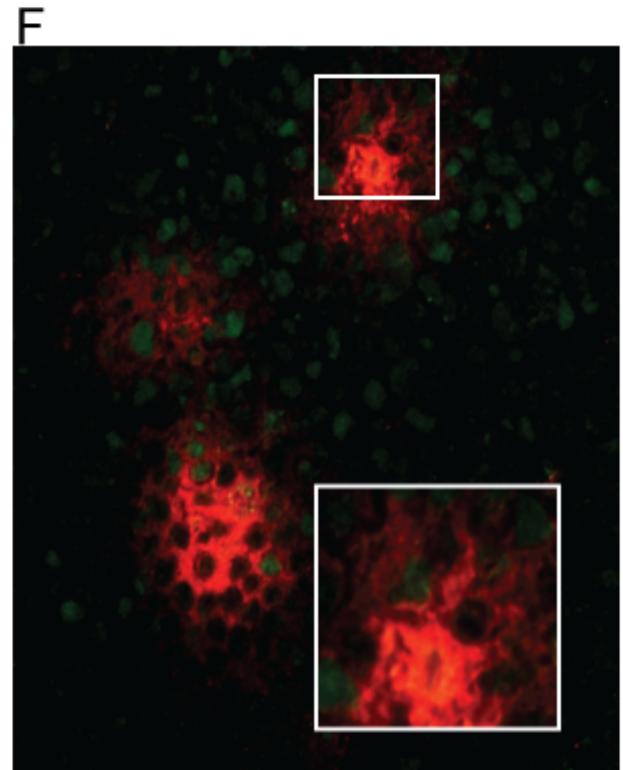

